\title{
Optimal Antenna Selection Designs for Coupled MIMO Systems
}

\author{
Pawandeep S. Taluja and Brian L. Hughes \\ Department of Electrical and Computer Engineering, North Carolina State University, Raleigh, NC 27695-7914, USA \\ Correspondence should be addressed to Pawandeep S. Taluja, pstaluja@ncsu.edu
}

Received 2 June 2011; Revised 16 September 2011; Accepted 17 September 2011

Academic Editor: Hon Tat Hui

Copyright ( 12012 P. S. Taluja and B. L. Hughes. This is an open access article distributed under the Creative Commons Attribution License, which permits unrestricted use, distribution, and reproduction in any medium, provided the original work is properly cited.

\begin{abstract}
We consider the impact of antenna mutual coupling on receive antenna selection systems. Prior work on selection with mutual coupling has not considered the effects of the inactive (i.e., unselected) antenna terminations and spatial noise correlation. In this work, we show that the presence of inactive antennas can profoundly alter system performance when the antennas are strongly coupled. We also propose a new antenna selection technique that seeks to exploit coupling to improve performance. Simulations suggest that the new technique can significantly outperform traditional selection when coupling is present.
\end{abstract}

\section{Introduction}

Multipath fading is known to deteriorate the performance of a wireless communication system. Spatial diversity techniques employing multiple-antenna systems at the receiver have been shown to combat fading and promise significant performance improvement over single-antenna systems [1, $2]$. The performance of such diversity techniques grows with the number of antennas though the gains gradually start to become less profound. Besides, multiple-input-multipleoutput (MIMO) architectures [3, 4] have been shown to increase the achievable data rate, tremendously.

However, these techniques demand deployment of large arrays, and, thus, the benefits come at the cost of an increased system and hardware complexity. At the receive side, each antenna is associated with a low-noise amplifier (LNA), a demodulator and an A/D converter, together constituting a receive radio frequency $(\mathrm{RF})$ chain, which is expensive. In addition to that, employing more antennas requires sophisticated digital signal processing, and, for small-sized or hand-held receivers, it puts a burden on providing an extended battery life. All these factors pose a challenge to the wide-scale deployment of multiple-antenna systems.

Antenna selection is one such scheme which tries to bridge the gap between the complexity and diversity benefits. It utilizes only a subset of available antenna signals, followed by down-conversion and digital signal processing. This reduces the requirement of a large number of $\mathrm{RF}$ chains and brings down the system and hardware complexity. An analysis of generalized diversity schemes with maximal-ratio combining (MRC) technique is reported in [5]. In this paper, we restrict our attention to receive antenna selection combined with MRC, also known as Hybrid-Selection/MRC (H-S/MRC) technique [6-9].

Although, MIMO techniques have been demonstrated to achieve the much needed high-throughput and reliable wireless communication, practical constraints on the physical dimensions of a transceiver limit the deployment of large arrays. Thus, in order to build low-cost and compact multiple-antenna devices, packing antennas closer together becomes indispensable.

As the antenna elements in an array are brought closer, the fading path gains become correlated, and the antennas begin to couple with one another. For closely spaced antennas, the current flowing in one element alters the voltage across the other, commonly known as mutual coupling. Traditionally, antenna selection applied to MIMO systems chooses a set active antennas to be employed for transmission or reception based on some performance metric and ignores the inactive antennas. However, in the case of closely spaced antennas, mutual coupling can have a profound impact on the performance, while also opening new avenues for transceiver design with respect to the presence of inactive elements in the vicinity of the active subset. 
It is well known that employing parasitic antennas in compact arrays can leverage significant performance improvements. In this paper, we investigate the design of compact receive antenna selection systems from a communication-theoretic perspective that put the inactive elements at use. The underlying motivation is to come up with selection architectures that offer improved diversity performance, compared to a system without selection with an equal number of RF chains and array size. An illustration of this comparison is given in Figure 1. It shows three systems with and without antenna selection, referred to as (a) antenna selection (AS), (b) reduced full-complexity (RFC), and (c) full-complexity (FC).

A study of receive antenna selection in the presence of mutual coupling has been reported in $[12,13]$. However, the performance of antenna selection applied to compact arrays is not well understood. These studies make differing assumptions about the role of inactive elements leading to differing conclusions. For example, the proposed model in [12] assumes that the inactive elements are transparent to the rest of the array at all spacings. While [13] has reported that, for compact arrays, the performance of antenna selection is worse than the full complexity system. It models the inactive elements as terminated in the same impedance as the active elements. However, we believe that terminating an inactive element in an impedance with nonzero resistive part would only degrade the system performance and thus, undermine the hidden potential of the inactive elements.

Besides, all studies, thus far, have assumed that the noise at the receiver is additive white Gaussian in nature. It has been recently shown in [14] that the noise for a coupled receiver is correlated in state-of-the-art compact receivers and that the performance is significantly altered by the exact location of the noise in the RF chain. We adopt the same spatially correlated noise model for the receiver.

As we present a more realistic model for receive antenna selection applied to compact arrays, we seek to address some of the above-mentioned issues. It will be shown in this paper that for compact arrays, selection is in fact preferred over compact full-complexity and reduced fullcomplexity systems. We shall demonstrate that, by appropriate termination of the inactive elements, the performance of antenna selection can be further improved. We call this strategy parasitic antenna selection (PAS). A simple selection technique with one active element will be shown to perform better than others employing more RF chains for certain configurations.

The organization of this paper is as follows. In Section 2, we discuss the impact of inactive elements in coupled systems. Section 3 introduces the generalized system model. Section 4 formulates the problem for a diversity system and discusses optimal matching and optimal/suboptimal parasitic networks for antenna selection. Section 5 outlines a numerical example and presents the results for various configurations. Section 8 highlights some of the implementation aspects of the proposed antenna selection scheme. Finally, we conclude the paper in Section 9.
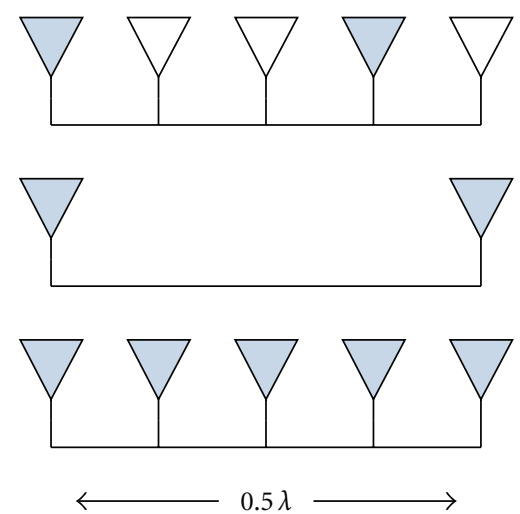

FIgURE 1: Configurations: (a) antenna selection (AS), (b) reduced full complexity (RFC), (c) full complexity (FC).

\section{Impact of Inactive Elements}

Traditional antenna selection schemes assume that the inactive elements are left open circuited. While the termination of inactive elements does not matter when the array elements are uncoupled, it is imperative to study the role played by the inactive elements in a compact selection system.

One such study that explores this aspect is [15]; wherein, the impact of an open-circuited antenna placed close to a driven half-wavelength dipole has been studied numerically. The two arms of the inactive element are treated as two quarter-wavelength shorted dipoles. Although, the terminal current in the open-circuited element is zero, the two arms carry a surface current due to coupling from the driven element. The results show that, for small spacings and practical thickness of these dipoles, the peak of this current distribution can be as large as $12 \%$ that of the current flowing in the driven element. These surface currents cause elements to reradiate incident EM fields, thereby not only affecting the open-circuit voltages across the active elements but also modifying the effective radiation pattern of the array. Clearly, the open-circuited inactive elements can no longer be treated as transparent to the rest of the array.

For long, inactive (aka parasitic) elements in an array of antennas have been exploited to improve system performance. For example, popular Yagi-Uda arrays [16] are known to provide powerful directivity. Such arrays are known to exploit the parasitic behavior in directional beamforming by optimal spacing, length, and short-circuiting of the inactive elements. However, these systems are sensitive to the direction of arrival of the signal.

Vaughan [10] has suggested that for a circular array of parasitic elements, with a single driven element in the center, it is possible to beamform the radiation by proper switching of parasites as open or short, but, to the best of our knowledge, such a scheme has not been proposed in conjunction with antenna selection. Hamer and Butcher [17] experimentally demonstrated a single switched-element design and investigated the impact of loading the inactive element. A similar study has been found in [18], where the author has considered a receive array with two dipoles-a fixed driven 


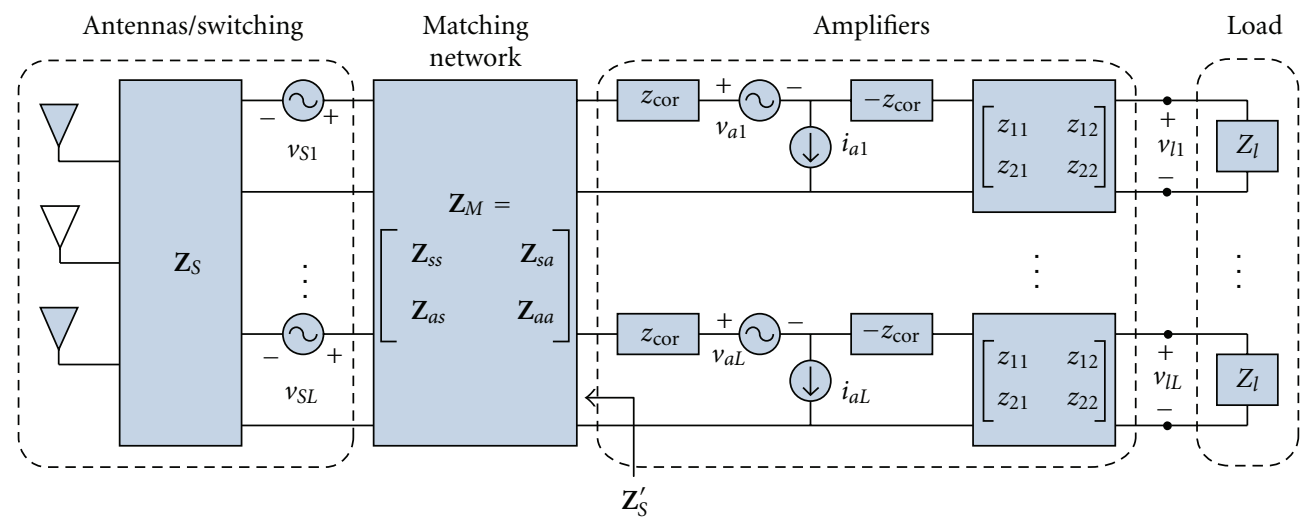

FIGURE 2: A circuit model of a receiver with antenna selection and mutual coupling.

element and a parasitic element loaded with an optimal impedance (such that the antenna correlation is minimized).

Harrington [11] has shown that it is possible to choose purely reactive loads for the nondriven elements of an array such that the overall radiation pattern can be steered over the azimuth. However, the techniques mentioned therein, require an exhaustive search for the optimal reactive load. Moreover, the loads are also a function of the direction of the steerable radiation pattern. When applied to the case of selection, it requires a different set of optimal reactive loads for each active subset and each channel realization. A popular flavor called electronically steerable passive array radiator (ESPAR) is applied to circular arrays with one active antenna centered in a ring of equidistant parasitic antennas with switchable reactive loads.

Hence, we seek to explore ways in which the coupling between the array elements can be exploited to improve the performance of selection systems. The key idea here is to use a different kind of termination that can reflect the power off of the inactive elements in order to make more power available to the active subset. In arriving at the analytically optimal designs, we shall begin with modeling the transceiver accurately.

\section{System Model}

We consider the receiver circuit model illustrated in Figure 2, which extends the model of [14] to include antenna selection. This model consists of an array of $N$ receive antennas together with a switch that selects a subset of these antennas to connect to $L$ RF chains. The switch is connected to the front-end amplifiers through a matching network, while the rest of the RF chain is represented by an equivalent load $z_{l}$. These components are described in detail below.

3.1. Antennas and Switching. The $N$ receive antennas convert the incident electromagnetic (EM) field into open-circuit voltages across the antenna terminals. When the antennas are closely spaced, the terminal voltage of each antenna depends not only on the field at that antenna but also on the currents flowing through neighboring antennas. To account for this coupling, the relationship between the terminal voltages $\mathbf{v}$ and currents $\mathbf{i}$ can be modeled as

$$
\mathbf{v}=\mathbf{Z}_{A} \mathbf{i}+\mathbf{v}_{o}
$$

where $\mathbf{Z}_{A}$ is an $N \times N$ impedance matrix and $\mathbf{v}_{o}$ is the $N \times 1$ open-circuit voltage induced by the electric field. Here $\left[\mathbf{Z}_{A}\right]_{n n}$ is the self-impedance of antenna $n$, and $\left[\mathbf{Z}_{A}\right]_{n m}$ is the mutual impedance between antennas $n$ and $m$. Approximate formulas for these impedances are given for a two-element array of infinitesimally thin dipoles in [19]. Since these formulas do not account for antenna scattering, we evaluate the impedances numerically for finite-width dipoles using the Numerical Electromagnetics Code (NEC) [20], a program based on the method of moments.

In a flat-fading environment, the open-circuit voltage in (1) is given by

$$
\mathbf{v}_{o}=\sqrt{\mathcal{E}} \mathbf{h}_{o} x+\mathbf{n}_{o}
$$

where $\mathbf{h}_{o}$ is an $N$-vector of fading path gains, $x$ is the complex transmitted symbol with $E\left[|x|^{2}\right]=1, E[\cdot]$ denotes the expectation, $\mathcal{E}$ is the average received energy per branch, and $\mathbf{n}_{o}$ is noise induced in the antennas. If the received signal field consists of a large number of plane waves with independent, uniformly-distributed random phases, then $\mathbf{h}_{o}$ can be modeled as a zero-mean, circularly symmetric, complex Gaussian random vector, denoted by $\mathbf{h}_{o} \sim \mathcal{C} \mathcal{N}\left(\mathbf{0}, \boldsymbol{\Sigma}_{\mathbf{h}_{o}}\right)$. For a uniform linear array with spacing $d$, the fading correlation matrix is $[21,22]$

$$
\begin{gathered}
{\left[\boldsymbol{\Sigma}_{\mathbf{h}_{o}}\right]_{n m}=\int_{0}^{2 \pi} \int_{0}^{\pi} g_{n}(\theta, \phi) g_{m}^{*}(\theta, \phi) e^{j(2 \pi / \lambda) d(n-m) \sin \theta \cos \phi}} \\
\times p(\theta, \phi) \sin \theta d \theta d \phi
\end{gathered}
$$

where $g_{n}(\theta, \phi)$ is the antenna pattern of dipole $n, p(\theta, \phi)$ is the angular density of multipaths arriving from $(\theta, \phi)$, and $\lambda$ is the wavelength. To account for antenna scattering, we calculate $g_{n}(\theta, \phi)$ numerically for finite-width dipoles using NEC. When multipaths are uniformly distributed in the plane $p(\theta, \phi)=(1 / 2 \pi) \delta(\theta-(\pi / 2))$, and scattering is 
neglected so each dipole is omnidirectional $g_{n}(\pi / 2, \phi)=$ 1 , note that (3) reduces to Clarke's [21, 22] formula $J_{0}(2 \pi(d / \lambda)(m-n))$.

For perfectly conducting antennas, $\mathbf{n}_{o}$ in (2) is the opencircuit voltage induced by noise sources in the surrounding environment, such as thermal radiation, cosmic background, and interference from other devices. As in [14], we assume that the array is surrounded by a black body enclosure at the standard temperature $T_{0}=290 \mathrm{~K}$. Under these conditions, Twiss [23] showed that $\mathbf{n}_{o} \sim \mathcal{C} \mathcal{N}\left(\mathbf{0}, \boldsymbol{\Sigma}_{\mathbf{n}_{o}}\right)$, where

$$
\Sigma_{\mathbf{n}_{o}}=2 k T_{0} B\left(\mathbf{Z}_{A}+\mathbf{Z}_{A}^{H}\right)
$$

$k=1.38 \times 10^{-23} \mathrm{~J} / \mathrm{K}$ is Boltzmann's constant, $H$ denotes the conjugate transpose, and $B$ is the bandwidth in Hz. Observe that $\mathbf{Z}_{A}$ is essentially diagonal for large $d$, so the noise is spatially white. For $d$ less than a few wavelengths, however, $\mathrm{Z}_{A}$ is not diagonal and the noise is correlated.

The array is followed by a noiseless switching network that selects $L$ out of $N$ "active" antennas to connect to the $L$ receiver RF chains. The remaining $\bar{L}=N-L$ "inactive" antennas are not connected directly to the receiver chains but, depending on how they are terminated, they can affect the active antennas through mutual coupling. Let $\delta=$ $\left\{s_{1}, \ldots, s_{L}\right\}$ denote the indices of the $L$ active antennas, and let $\bar{\delta}=\left\{\bar{s}_{1}, \ldots, \bar{s}_{N-L}\right\}$ be the corresponding indices of the $\bar{L}$ inactive antennas. As illustrated in Figure 3, it is convenient to think of the switch as partitioning the antennas into an active group with open-circuit voltage, $\mathbf{v}_{o}(\delta)=\mathbf{I}_{\delta} \mathbf{v}_{o}$, and an inactive group with open-circuit voltage $\mathbf{v}_{o}(\bar{\delta})=\mathbf{I} \bar{\delta} \mathbf{v}_{o}$, where $\mathbf{I}_{\S}$ is the $|\&| \times N$ selection matrix defined by

$$
\left[\mathbf{I}_{s}\right]_{i j}= \begin{cases}1, & \text { if } j=s_{i} \\ 0, & \text { otherwise }\end{cases}
$$

and $|\&|$ is the cardinality of $\&$.

In traditional antenna selection, the inactive antennas are considered to be open circuit. Here we also consider the possibility that terminating the inactive antennas with some $\bar{L} \times \bar{L}$ parasitic impedance network, say $\mathbf{Z}_{P}$, may improve performance. Since this impedance may allow currents to flow through the inactive elements, it may alter the opencircuit voltage and impedance of the active antennas in a way that depends on $\$$. If we partition (1) as in Figure 3, we obtain

$$
\left[\begin{array}{l}
\mathbf{v}(\bar{\delta}) \\
\mathbf{v}(\delta)
\end{array}\right]=\left[\begin{array}{ll}
\mathbf{Z}_{\bar{\delta}} & \mathbf{Z}_{\bar{\delta}} \\
\mathbf{Z}_{\delta \bar{\delta}} & \mathbf{Z}_{\S \S}
\end{array}\right]\left[\begin{array}{l}
\mathbf{i}(\bar{\delta}) \\
\mathbf{i}(\delta)
\end{array}\right]+\left[\begin{array}{l}
\mathbf{v}_{o}(\bar{\delta}) \\
\mathbf{v}_{o}(\delta)
\end{array}\right],
$$

where $\mathbf{v}(\S)=\mathbf{I}_{\S} \mathbf{v}, \mathbf{i}(\S)=\mathbf{I}_{\S} \mathbf{i}, \mathbf{Z}_{\S \bar{\delta}}=\mathbf{I}_{\S} \mathbf{Z}_{A} \mathbf{I}_{\S}$, and the other quantities are defined analogously. Here, $T$ denotes the transpose. Note we also have

$$
\mathbf{v}(\overline{8})=-\mathbf{Z}_{P} \mathbf{i}(\overline{8})
$$

Combining (6) and (7), we obtain

$$
\begin{aligned}
& \mathbf{i}(\bar{\delta})=-\left(\mathbf{Z}_{P}+\mathbf{Z}_{\bar{\delta} \bar{\delta}}\right)^{-1}\left(\mathbf{Z}_{\bar{\delta} \delta} \mathbf{i}(\delta)+\mathbf{v}_{o}(\bar{\delta})\right), \\
& \mathbf{v}(\S)=\mathbf{Z}_{\delta} \mathbf{i}(\S)+\mathbf{v}_{\S},
\end{aligned}
$$

where

$$
\begin{aligned}
& \mathbf{Z}_{\S}=\mathbf{Z}_{\S \S}-\mathbf{Z}_{\S \bar{\S}}\left(\mathbf{Z}_{P}+\mathbf{Z}_{\bar{\S} \bar{\S}}\right)^{-1} \mathbf{Z}_{\bar{\S} \S}, \\
& \mathbf{v}_{\S}=\mathbf{v}_{o}(\S)-\mathbf{Z}_{\S \bar{\zeta}}\left(\mathbf{Z}_{P}+\mathbf{Z}_{\bar{\delta} \bar{\delta}}\right)^{-1} \mathbf{v}_{o}(\bar{\delta}) \text {. }
\end{aligned}
$$

Comparing (8) with (1), we see that the switching network and $\mathbf{Z}_{P}$ essentially change the array impedance and opencircuit voltage from $\mathbf{Z}_{A}$ and $\mathbf{v}_{o}$ to $\mathbf{Z}_{\S}$ and $\mathbf{v}_{\S}$, respectively.

Most of the voltages and currents in Figure 2 will, like those in (8), (9), and (10), depend on the set of active antennas, \&. However, to avoid needless clutter, we have chosen to suppress this dependence in (9) and (10). We also note for future use that $(10)$ can be written as

$$
\mathbf{v}_{\S}=\mathbf{T}_{\S} \mathbf{v}_{o} \mathbf{T}_{\S}=\mathbf{I}_{\S}-\mathbf{Z}_{\S \bar{\delta}}\left(\mathbf{Z}_{P}+\mathbf{Z}_{\bar{\delta} \bar{\delta}}\right)^{-1} \mathbf{I}_{\bar{\delta}}
$$

where $\mathbf{T}_{\S}$ is the equivalent selection matrix.

3.2. Amplifiers. According to [24], any linear amplifier can be represented as in Figure 2. Here,

$$
v_{a 1} \sim \mathcal{C} \mathcal{N}\left(0,4 k T_{0} B r_{a}\right), \quad i_{a 1} \sim \mathcal{C} \mathcal{N}\left(0,4 k T_{0} B g_{a}\right)
$$

are independent random variables that model amplifier internal noise, where $r_{a}$ and $g_{a}$ are the equivalent noise resistance and equivalent noise conductance, respectively. The correlation impedance, $z_{\text {cor }}=r_{\text {cor }}+j x_{\text {cor }}$, controls the correlation between the noise observed at the two output terminals. The noise statistics of an amplifier are completely characterized by $\left\{r_{a}, g_{a}, z_{\text {cor }}\right\}$.

When a source impedance $z_{s}=r_{s}+j x_{s}$ at the standard temperature is connected to the amplifier input port, the noise factor is defined as

$$
F=1+\frac{1}{r_{s}}\left(r_{a}+g_{a}\left|z_{s}+z_{\mathrm{cor}}\right|^{2}\right),
$$

The noise factor is useful because it relates the input and output signal-to-noise ratios (SNRs) of the amplifier, given in $\mathrm{dB}$ by $\mathrm{SNR}_{\text {out }}=\mathrm{SNR}_{\text {in }}-\mathrm{NF}$, where $\mathrm{NF}=10 \log _{10} F$ is the noise figure. Note that $F$ attains its minimum value $F_{\min }$ when $z_{s}=\mathrm{z}_{\mathrm{opt}}$ where [24]

$$
\begin{gathered}
F_{\mathrm{min}}=1+2\left(g_{a} r_{\mathrm{cor}}+\sqrt{g_{a} r_{a}+\left(g_{a} r_{\mathrm{cor}}\right)^{2}}\right), \\
z_{\mathrm{opt}}=\sqrt{\frac{r_{a}}{g_{a}}+r_{\mathrm{cor}}^{2}}-j x_{\mathrm{cor} .}
\end{gathered}
$$

3.3. Matching Network. The switching network is connected to the amplifiers by a $2 L$-port-matching network $\mathbf{Z}_{M}$. Matching networks can be used to alter the antenna array impedance in order to maximize the power transfer or the resulting SNR. We assume an ideal matching network consisting of passive and reactive elements such that it is noiseless, lossless, and reciprocal. It can be shown [25] that the network is lossless (no power is dissipated within it) provided that the following conditions are satisfied: $\mathbf{Z}_{a a}=$ $-\mathbf{Z}_{a a}^{H}, \mathbf{Z}_{s s}=-\mathbf{Z}_{s s}^{H}$, and $\mathbf{Z}_{s a}=-\mathbf{Z}_{a s}^{H}$. 


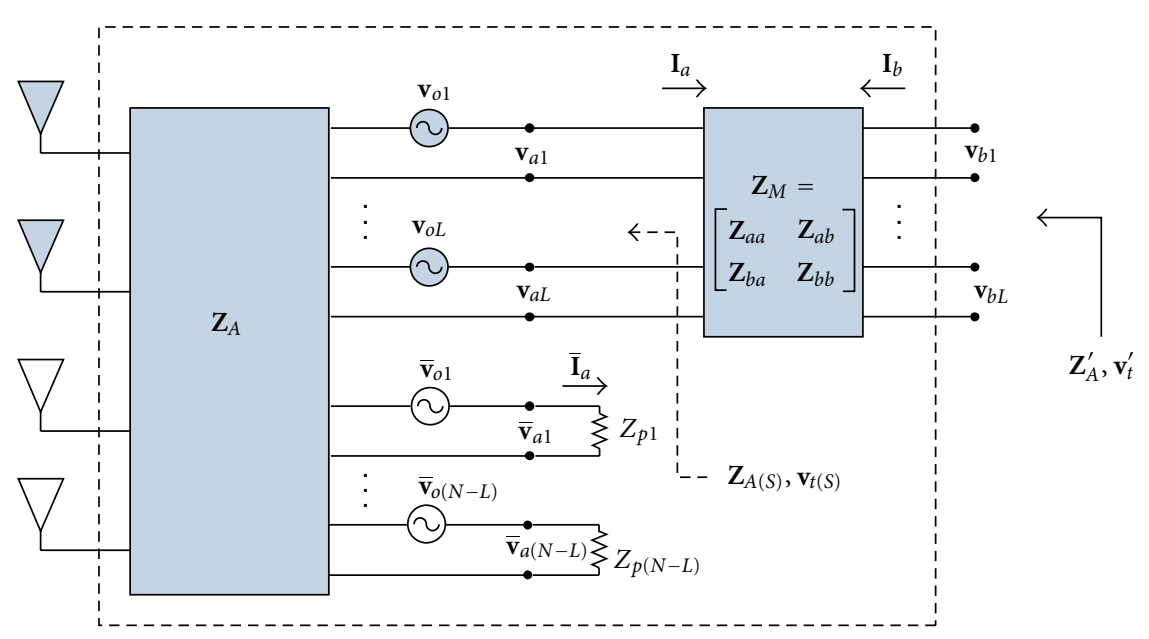

FIgURE 3: Circuit model of the antenna array and switching network.

The relationship between the voltages and currents at the input and output of the matching network are described by equations similar to (6) and (7), with $\mathbf{Z}_{P}$ replaced with $\mathbf{Z}_{\S}, \mathbf{v}_{o}(\bar{\delta})$ replaced with $-\mathbf{v}_{\S}, \mathbf{v}_{o}(\S)$ replaced with $\mathbf{0}, \mathbf{Z}_{a s}$ replaced with $\mathbf{Z}_{\S \bar{s}}$, and so on. It follows from (8) that the matching network alters the switch impedance and opencircuit voltage from $\mathbf{Z}_{\S}$ and $\mathbf{v}_{\S}$ to $\mathbf{Z}_{\S}^{\prime}$ and $\mathbf{v}_{\S}^{\prime}$, respectively, where

$$
\begin{gathered}
\mathbf{Z}_{\S}^{\prime}=\mathbf{Z}_{a a}-\mathbf{Z}_{a s}\left(\mathbf{Z}_{\S}+\mathbf{Z}_{s s}\right)^{-1} \mathbf{Z}_{s a}, \\
\mathbf{v}_{\S}^{\prime}=\mathbf{Z}_{a s}\left(\mathbf{Z}_{\S}+\mathbf{Z}_{s s}\right)^{-1} \mathbf{v}_{\S} .
\end{gathered}
$$

3.4. Load. The rest of the RF chain (aka downstream components) is modeled by an Thevenin equivalent load. These elements typically consist of demodulation components like mixers, filters, and samplers.

\section{Optimal Designs for Diversity System}

The input to the linear combiner given by the voltage $\mathbf{v}_{l}$ across the load $\mathbf{z}_{l}$ can be expressed in terms of the $L \times 1$ equivalent fading path-gain vector $\mathbf{h}$ as

$$
\mathbf{r}=\mathbf{v}_{l}=\sqrt{\mathcal{E}} \mathbf{h} x+\mathbf{n},
$$

where

$$
\begin{gathered}
\mathbf{h}=\operatorname{DCM}\left(\mathbf{T}_{\S} \mathbf{h}_{o}\right), \\
\mathbf{n}=\operatorname{DC}\left[\mathbf{M}\left(\mathbf{T}_{\S} \mathbf{n}_{o}\right)-\mathbf{v}_{a}-\left(\mathbf{Z}_{\S}^{\prime}+z_{\mathrm{cor}} \mathbf{I}\right) \mathbf{i}_{a}\right] .
\end{gathered}
$$

Here, $\mathbf{v}_{a}$ and $\mathbf{i}_{a}$ are the amplifier noise voltages and currents, respectively, and

$$
\begin{gathered}
\mathbf{C}=z_{21}\left(\mathbf{Z}_{\S}^{\prime}+z_{11} \mathbf{I}\right)^{-1}, \\
\mathbf{D}=z_{l}\left[\left(z_{l}+z_{22} \mathbf{I}\right)-z_{12} \mathbf{C}\right]^{-1}, \\
\mathbf{M}=\mathbf{Z}_{a s}\left(\mathbf{Z}_{\S}+\mathbf{Z}_{s s}\right)^{-1},
\end{gathered}
$$

with $\mathbf{Z}_{\text {\& }}^{\prime}$ given by (15).
The amplifier parameters are denoted by $z_{11}, z_{12}, z_{21}$ and $z_{22}$ and the mutually independent noise sources are distributed as $\mathbf{v}_{a} \sim \mathcal{C} \mathcal{N}\left(\mathbf{0}, 4 k T_{0} B r_{a} \mathbf{I}\right)$ and $\mathbf{i}_{a} \sim$ $\mathcal{C} \mathcal{N}\left(\mathbf{0}, 4 k T_{0} B g_{a} \mathbf{I}\right)$. The noise covariance $\boldsymbol{\Sigma}_{\mathbf{n}}$ for (19) is, thus, given by

$$
\begin{aligned}
\frac{1}{4 k T_{0} B} \boldsymbol{\Sigma}_{\mathbf{n}}= & \mathrm{DC}\left[\frac{1}{2} \boldsymbol{\Sigma}_{\mathbf{n}_{0}}^{\prime}+r_{a} \mathbf{I}+g_{a}\left(\mathbf{Z}_{S}^{\prime}+z_{\mathrm{cor}} \mathbf{I}\right)\left(\mathbf{Z}_{S}^{\prime}+z_{\mathrm{cor}} \mathbf{I}\right)^{H}\right] \\
& \times C^{H} D^{H},
\end{aligned}
$$

where $\boldsymbol{\Sigma}_{\mathbf{n}_{o}}^{\prime}=\left(\mathbf{M T}_{\S}\right) \boldsymbol{\Sigma}_{\mathbf{n}_{o}}\left(\mathbf{M T}_{\S}\right)^{H}$.

4.1. Optimal Combining. The receiver employs a linear combiner to the input $\mathbf{r}$ such that

$$
y=\mathbf{w}^{H} \mathbf{r}=\hat{h} x+\hat{n},
$$

and the resulting SNR is

$$
\gamma=\frac{|\hat{h}|^{2}}{\mathrm{E}\left[|\hat{n}|^{2}\right]}=\mathcal{E} \frac{\left|\mathbf{w}^{H} \mathbf{h}\right|^{2}}{\mathbf{w}^{H} \boldsymbol{\Sigma}_{\mathbf{n}} \mathbf{w}} .
$$

The well-known optimal maximal-ratio-combiner (MRC) is given by $\mathbf{w} \propto \boldsymbol{\Sigma}_{\mathbf{n}}^{-1 / 2} \mathbf{h}$. Thus, the optimal SNR for the antenna selection system becomes

$$
\gamma^{o}=\mathcal{E}_{\mathbf{I}_{\delta}}\left\|\boldsymbol{\Sigma}_{\mathbf{n}}^{-1 / 2} \mathbf{h}\right\|^{2}
$$

where $\mathbf{h}$ is given by (18) and $\boldsymbol{\Sigma}_{\mathbf{n}}$ by (21).

4.2. Optimal Matching Network. For a given $\&$, any matching network that results in the impedance $\mathbf{Z}_{s}^{\prime}=z_{\mathrm{opt}} \mathbf{I}$ minimizes the outage probability of selection diversity [26]. Here, $z_{\mathrm{opt}}$ is the source impedance (14) that achieves the minimum noise 
factor of the amplifier. One lossless, reciprocal network that accomplishes this task is [14]

$$
\begin{aligned}
\mathbf{Z}_{M} & =\left[\begin{array}{ll}
\mathbf{Z}_{s s} & \mathbf{Z}_{s a} \\
\mathbf{Z}_{a s} & \mathbf{Z}_{a a}
\end{array}\right] \\
& =j\left[\begin{array}{cc}
-\operatorname{Im}\left[\mathbf{Z}_{\S}\right] & \left(r_{\mathrm{opt}} \operatorname{Re}\left[\mathbf{Z}_{\S}\right]\right)^{1 / 2} \\
\left(r_{\mathrm{opt}} \operatorname{Re}\left[\mathbf{Z}_{\S}\right]\right)^{1 / 2} & x_{\mathrm{opt}} \mathbf{I}
\end{array}\right] .
\end{aligned}
$$

While optimal, this network would not be easy to realize in practice. The dependence on $\&$ would generally require some kind of adaptive multiport matching, which would be a complex choice for a diversity scheme whose primary value is simplicity and economy. In some cases (e.g., when inactive antennas are open circuit), it is clear that $\mathbf{Z}_{\delta}^{\prime}=z_{\text {opt }} \mathbf{I}$ could also be achieved by a fixed matching network located between the antennas and the switch. In any case, we consider (25) here primarily because it provides an upper bound on the performance of any fixed matching network.

We also consider a simpler, suboptimum matching strategy that applies to each antenna the two-port matching network that achieves the minimum noise figure for that antenna in isolation. This is called self-matching for minimum noise figure and is accomplished by replacing $\mathbf{Z}_{\&}$ with $z_{A} \mathbf{I}$ in (25), where $z_{A}=r_{A}+j x_{A}$ is the self-impedance of each antenna in isolation

$$
\mathbf{Z}_{M}=j\left[\begin{array}{cc}
-x_{A} \mathbf{I} & \sqrt{r_{\mathrm{opt}} r_{A}} \mathbf{I} \\
\sqrt{r_{\mathrm{opt}} r_{A}} \mathbf{I} & x_{\mathrm{opt}} \mathbf{I}
\end{array}\right] .
$$

4.3. Optimal Parasitic Network. Observe that the optimal matching network discussed above depends on (9) which in turn is also dependent on the parasitic network. Hence, it is natural to ask if there is an optimal choice for the parasitic networks (with purely reactive entries) as well. The aim here is to choose a $\mathbf{Z}_{P}$ that depends only on the active subset $\&$ and not on the instantaneous channel conditions, just like the optimal matching network $\mathbf{Z}_{M}$.

The derivation of optimal nondiagonal $\mathbf{Z}_{P}$ for a uniform linear array is in general complicated and tedious. However, the solution is analytically tractable for a special but practical class of planar arrays_-uniform circular arrays - with equal number of active and inactive elements. Again, the optimal parasitic network design presented here serves as an upper bound on the system performance of coupled antenna selection systems.

To that end, consider a circular array of $N$ antennas such

$$
L=\bar{L}=N-L .
$$

The impedance matrix $\mathbf{Z}_{A}$ for a circular array is circulant, and the same applies to the permuted impedance matrix in (6)

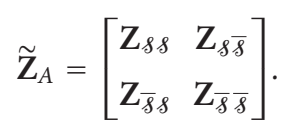

Each of the $L \times L$ subblocks in (28) can be diagonalized by a unitary transformation $\mathbf{Q}$ such that

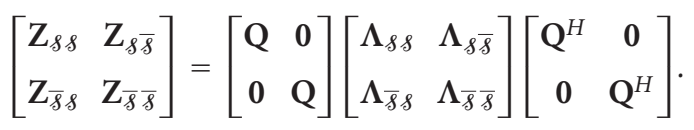

The set of orthonormal eigen vectors for each block corresponding to the eigen-values $\Lambda$ are given by

$$
\mathbf{Q}=\frac{1}{\sqrt{L}}\left[\begin{array}{ccccc}
1 & 1 & 1 & \ldots & 1 \\
1 & \omega & \omega^{2} & \ldots & \omega^{L-1} \\
1 & \omega^{2} & \omega^{4} & \ldots & \omega^{2(L-1)} \\
\vdots & \vdots & \vdots & & \vdots \\
1 & \omega^{L-1} & \omega^{2(L-1)} & \ldots & \omega^{(L-1)(L-1)}
\end{array}\right]
$$

where $\omega=e^{-j 2 \pi / L}$ and the eigen-values $(\boldsymbol{\Lambda})$ are given by the DFT of the first row scaled by $\sqrt{L}$. Physically, the matrix $\mathbf{Q}$ represents an $L$-point DFT/IDFT operation in space.

Since the subblocks of $\mathbf{Z}_{A}$ are $L \times L$ circulant matrices, it is reasonable to assume, as is also evident from (9), that the optimal parasitic network also admits the same spatial unitary transformation $\mathbf{Q}$ diagonalizing it

$$
\mathbf{Z}_{P}=\mathbf{Q} \Lambda_{P} \mathbf{Q}^{H} .
$$

Therefore, we can write (9) as

$$
\mathbf{Z}_{\delta}=\underbrace{\left.\mathbf{Q} \Lambda_{\delta \&}-\Lambda_{\delta \bar{\delta}}\left(\Lambda_{P}+\Lambda_{\bar{\delta} \bar{\delta}}\right)^{-1} \Lambda_{\bar{\delta}}\right)}_{\Lambda_{\delta}} \mathbf{Q}^{H}
$$

such that the optimal matching network from (25) is given by

$$
\begin{aligned}
\mathbf{Z}_{M} & =\left[\begin{array}{ll}
\mathbf{Z}_{s s} & \mathbf{Z}_{s a} \\
\mathbf{Z}_{a s} & \mathbf{Z}_{a a}
\end{array}\right] \\
& =\left[\begin{array}{ll}
\mathbf{Q} & \mathbf{0} \\
\mathbf{0} & \mathbf{Q}
\end{array}\right]\left[\begin{array}{ll}
\boldsymbol{\Lambda}_{s s} & \boldsymbol{\Lambda}_{s a} \\
\boldsymbol{\Lambda}_{a s} & \boldsymbol{\Lambda}_{a a}
\end{array}\right]\left[\begin{array}{cc}
\mathbf{Q}^{H} & \mathbf{0} \\
\mathbf{0} & \mathbf{Q}^{H}
\end{array}\right],
\end{aligned}
$$

where

$$
\left[\begin{array}{ll}
\boldsymbol{\Lambda}_{s s} & \boldsymbol{\Lambda}_{s a} \\
\boldsymbol{\Lambda}_{a s} & \boldsymbol{\Lambda}_{a a}
\end{array}\right]=j\left[\begin{array}{cc}
-\operatorname{Im}\left[\boldsymbol{\Lambda}_{\delta}\right] & \left(r_{o} \operatorname{Re}\left[\boldsymbol{\Lambda}_{\delta}\right]\right)^{1 / 2} \\
\left(r_{o} \operatorname{Re}\left[\boldsymbol{\Lambda}_{\delta}\right]\right)^{1 / 2} & x_{o} \mathbf{I}
\end{array}\right] .
$$

The input impedance seen looking into the matching network from the rest of the RF chain is given by(15)

$$
\mathbf{Z}_{s}^{\prime}=\mathbf{Q} \underbrace{\left(\Lambda_{a a}-\Lambda_{a s}\left(\Lambda_{s}+\Lambda_{s s}\right)^{-1} \Lambda_{s a}\right)}_{\Lambda_{s}^{\prime}} \mathbf{Q}^{H} .
$$

However, we can simplify the network design above by embedding special RF networks popularly called Butler matrices, that behave as spatial DFT/IDFT matrices, followed by a bank of uncoupled matching networks, that is, $\mathbf{Z}_{M}=\boldsymbol{\Lambda}_{M}$ 


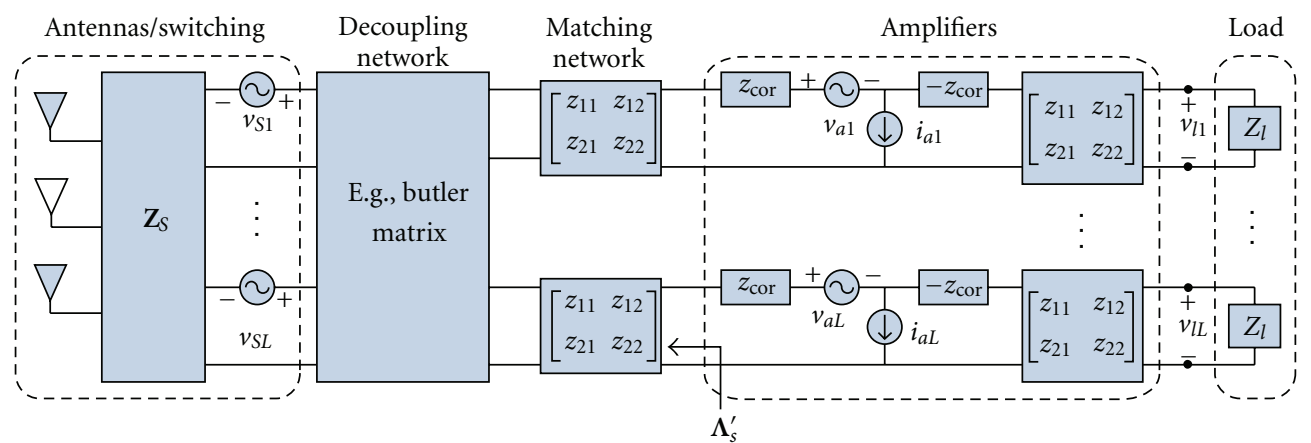

FIGURE 4: A circuit model of a circular array receiver with antenna selection and mutual coupling.

(as shown in Figure 4). The cascade of decoupling network and matching network can be expressed as

$$
\mathbf{Z}_{C}=\left[\begin{array}{ll}
\mathbf{Q} & \mathbf{0} \\
\mathbf{0} & \mathbf{I}
\end{array}\right]\left[\begin{array}{cc}
\boldsymbol{\Lambda}_{s s} & \boldsymbol{\Lambda}_{s a} \\
\boldsymbol{\Lambda}_{a s} & \boldsymbol{\Lambda}_{a a}
\end{array}\right]\left[\begin{array}{cc}
\mathbf{Q}^{H} & \mathbf{0} \\
\mathbf{0} & \mathbf{I}
\end{array}\right]
$$

such that the input impedance $\mathbf{Z}_{\S}^{\prime}$ becomes uncoupled

$$
\mathbf{Z}_{\S}^{\prime}=\Lambda_{a a}-\Lambda_{a s}\left(\Lambda_{s}+\Lambda_{s s}\right)^{-1} \Lambda_{s a}=\Lambda_{\S}^{\prime} .
$$

The post-MRC output SNR for amplifier-noisedominant scenarios with optimal parasitic network given by (24) is

$$
\begin{aligned}
\gamma^{o}\left(\boldsymbol{\Lambda}_{P}\right) & =\mathcal{E}_{\delta} \max _{\delta}\left\{\max _{\boldsymbol{\Lambda}_{P}(\delta)}\left\|\boldsymbol{\Sigma}_{\mathbf{n}}^{-1 / 2} \mathbf{h}\right\|^{2}\right\} \\
& =\frac{\mathcal{E}}{\left|\sigma_{a}\right|^{2}} \max _{\delta}\left\{\max _{\boldsymbol{\Lambda}_{P}(\delta)} \Gamma\left(\boldsymbol{\Lambda}_{P}(\delta)\right)\right\},
\end{aligned}
$$

where $\left|\sigma_{a}\right|^{2}=r_{a}+g_{a}\left|z_{o}+z_{\text {cor }}\right|^{2}$. The instantaneous post-MRC output SNR for each subset is given by

$$
\Gamma\left(\boldsymbol{\Lambda}_{P}(\&)\right)=\left\|\boldsymbol{\Lambda}_{M}^{H} \mathbf{h}^{\prime}\right\|^{2},
$$

where $\mathbf{h}^{\prime}=\mathbf{Q}^{H} \mathbf{T}_{\S} \mathbf{h}_{o}$ and

$$
\boldsymbol{\Lambda}_{M}=\sqrt{r_{o}}\left(\operatorname{Re}\left[\boldsymbol{\Lambda}_{\S \S}-\boldsymbol{\Lambda}_{\S \bar{s}}\left(\boldsymbol{\Lambda}_{P}+\boldsymbol{\Lambda}_{\bar{\zeta} \bar{\delta}}\right)^{-1} \boldsymbol{\Lambda}_{\bar{\delta} \delta}\right]\right)^{-1 / 2} .
$$

For details, see Appendix A.

The fading-independent, subset-dependent optimal $\Lambda_{P}$ is thus given by

$$
\begin{aligned}
\boldsymbol{\Lambda}_{P} & =\arg _{\boldsymbol{\Lambda}_{P}} \max \left\{\mathrm{E}\left(\Gamma\left(\boldsymbol{\Lambda}_{P}\right)\right)\right\} \\
& =\arg _{\boldsymbol{\Lambda}_{\mathrm{P}}} \max \left\{\mathrm{E}\left(\sum_{k=1}^{L}\left|\left[\lambda_{M}\right]_{k} h_{k}^{\prime}\right|^{2}\right)\right\} \\
& =\arg _{\boldsymbol{\Lambda}_{P}} \max \left\{\sum_{k=1}^{L}\left|\left[\lambda_{M}\right]_{k}\right|^{2} \mathrm{E}\left(\left|h_{k}^{\prime}\right|^{2}\right)\right\} .
\end{aligned}
$$

For a circular array with $N=4, L=2$

$$
\mathbf{Q}=\frac{1}{\sqrt{2}}\left[\begin{array}{cc}
1 & 1 \\
1 & -1
\end{array}\right] \text {. }
$$

Table 1: Optimal Parasitic Network: Circular Array $N=4, L=$ $2, d=0.1 \sqrt{2} \lambda$.

\begin{tabular}{lcc}
\hline Active Subset & Parameter & Value \\
\hline \multirow{2}{*}{$(1,3)$ or $(2,4)$} & {$\left[\boldsymbol{\Lambda}_{P}\right]_{1}$} & $-j 21.8894$ \\
& {$\left[\boldsymbol{\Lambda}_{P}\right]_{2}$} & Any \\
\hline \multirow{2}{*}{ Others } & {$\left[\boldsymbol{\Lambda}_{P}\right]_{1}$} & $-j 47.2350$ \\
& {$\left[\boldsymbol{\Lambda}_{P}\right]_{2}$} & $-j 20.5069$ \\
\hline
\end{tabular}

The optimal eigen values of $\mathbf{Z}_{P}$ are shown in Table 1 which are computed as the values that maximize a polynomial of degree two. Detailed information can be found in Appendix B.

The optimal parasitic network designs presented here for circular arrays with equal number of active and inactive elements can be extended to a broader set of arrays and configurations although the optimal solution may need to be computed numerically. In Section 7 , we shall provide some suboptimal designs applicable to all kinds of arrays and configurations which will be shown to achieve near-optimal performance for linear as well as circular arrays.

\section{Simulation}

In this section, we present numerical results for the two types of antenna arrays considered above. These arrays consist of half-wavelength dipole antennas spread over a length $L_{r}=$ $0.5 \lambda$ (for ULA) and a diameter of $D_{r}=0.3 \lambda$ (for UCA), as shown in Figure 5. The aim is to determine if employing antenna selection with closely packed antennas can provide benefits over conventional diversity systems in the presence of moderate to strong coupling.

We compare three combinations (a), (b), and (c), as shown in Figure 1, with and without antenna selection. Monte-Carlo simulations are carried out over 100, 000 channel realizations. The channel is assumed to be quasistatic block fading such that the block length is long enough to determine the optimal subset and parasitic network. The optimal combination, once determined, is employed for the rest of the block.

The closed form expression for the fading path-gain covariance given in $[21,22]$ assumes a uniform distribution 
of infinite EM waves arriving at the antenna, in the azimuth plane. While this may provide accurate values for thin dipole antennas where the active and inactive antennas do not act as scatterers, we resort to the use of NEC to account for the finite thickness (radius $100 \mathrm{~mm}$ ) of half-wavelength dipoles, and the scattering arising due to the presence of neighboring closely spaced elements. For similar reasons, we choose NEC for computing $\mathbf{Z}_{A}$ over the closed-form expressions for self and mutual impedance of the array of dipoles, as given in [19].

For an $N$ dipole-antenna uniform linear array with interelement spacing $d$, the incident electric field is modeled in NEC as a superposition of $M=32$ vertically polarized plane waves with i.i.d. phases uniformly distributed on $[0,2 \pi)$. The angles-of-arrival (AOA) of the plane waves, $\phi_{0}, \ldots, \phi_{M-1}$, are uniformly spaced in azimuth from 0 to $2 \pi$. Under these conditions, the open-circuit fading path gains are approximately Gaussian with correlation matrix

$$
\left[\boldsymbol{\Sigma}_{\mathbf{h}}\right]_{n m}=\sum_{k=0}^{M-1} g_{n}\left(\phi_{k}\right) g_{m}^{*}\left(\phi_{k}\right) e^{j 2 \pi(n-m) d / \lambda \cos \left(\phi_{k}\right)},
$$

where $g_{n}(\phi)$ is the open-circuit voltage induced in the $n$th antenna by a zero-phase plane wave with AOA $\phi$, normalized so that $\sum_{k}\left|g_{n}\left(\phi_{k}\right)\right|^{2}=1$ for an isolated dipole. For a uniform circular array, the correlation entries are given by

$$
\left[\boldsymbol{\Sigma}_{\mathbf{h}}\right]_{n m}=\sum_{k=0}^{M-1} g_{n}\left(\phi_{k}\right) g_{m}^{*}\left(\phi_{k}\right) e^{j 2 \pi d_{n m} \cos \left(\phi_{k}\right) / \lambda} .
$$

The amplifier selected for this study is a low-cost SiGe LNA [27] designed for use in the cellular band. In high-gain mode with $R_{\text {bias }}=510 \Omega$ and $f=900 \mathrm{MHz}$, its impedance matrix and the noise parameters are

$$
\left[\begin{array}{ll}
z_{11} & z_{12} \\
z_{21} & z_{22}
\end{array}\right]=\left[\begin{array}{cc}
35.7 \angle-82.0^{\circ} & 2.74 \angle 91.8^{\circ} \\
325 \angle 119^{\circ} & 46.1 \angle-23.3^{\circ}
\end{array}\right] \Omega
$$

$r_{a}=9.45 \Omega, g_{a}=3.24 \mathrm{mS}, z_{\text {cor }}=35.3 \angle-114$. We assume that the downstream noise is negligible compared to other noise sources [14].

It is worthwhile to note that according to the model used in earlier studies, $[12,13]$, the equivalent channel vector is given by

$$
\begin{gathered}
\tilde{\mathbf{h}}=\operatorname{DCM}\left(\mathbf{I}_{\delta} \Sigma_{\mathbf{h}_{o}}^{1 / 2} \mathbf{I}_{\delta}^{T}\right)\left(\mathbf{I}_{\delta} \mathbf{h}_{w}\right), \\
\hat{\mathbf{h}}=\mathbf{I}_{s}\left(\mathbf{D C M h}_{o}\right),
\end{gathered}
$$

respectively. Here, D, C, $\mathbf{M}$ are the corresponding $L \times L$ and $N \times N$ matrices, for $\widetilde{\mathbf{h}}$ and $\hat{\mathbf{h}}$, respectively. We point out that although the model considered in these studies does not include amplifiers, and matching is handled by way of altering the load, these equations still retain the essence of inactive-element modeling. We shall use these expressions to compare our results, whereas our model (c.f. (18)) suggests that it suffices to capture the accurate open circuit voltage across the active antennas, by taking into account the effect of inactive elements.
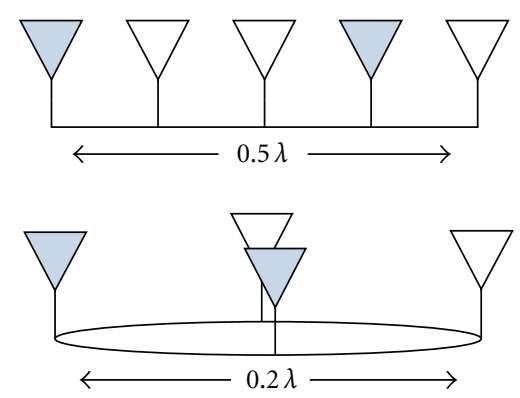

FIGURE 5: Configurations: (a) uniform linear array, (b) uniform circular array.

Before presenting the results, it is useful to normalize (24), such that for the i.i.d. scenario, the SNR is given by the familiar expression

$$
\gamma_{n}^{\mathrm{iid}}=\frac{\mathcal{E}}{N_{0}} \max _{\mathbf{I}_{\S}}\left\|\mathbf{I}_{\S} \mathbf{h}_{w}\right\|^{2},
$$

where $N_{0}$ represents the i.i.d. noise covariance for a single branch $\left(\boldsymbol{\Sigma}_{\mathbf{n}}=N_{0} \mathbf{I}\right)$, and $\mathbf{h}_{w}$ is the $N \times 1$ i.i.d. fading pathgain vector given by $\mathbf{h}_{w} \sim \mathcal{C} \mathcal{N}(\mathbf{0}, \mathbf{I})$.

To that end, consider a sufficiently large antenna spacing such that $\boldsymbol{\Sigma}_{\mathbf{h}}=\mathbf{I}$, and the antenna array is uncoupled, that is, $\mathbf{Z}_{A}=z_{A} \mathbf{I}$. From (11), $\mathbf{T}_{\S}=\mathbf{I}_{\&}$ and from (21), we have

$$
\Sigma_{\mathbf{n}}^{\mathrm{iid}}=4 k T_{0} B \sigma^{2} \mathbf{I},
$$

where

$$
\begin{gathered}
\sigma^{2}=\left(r_{\mathrm{opt}}+\sigma_{a}^{2}\right)|\alpha \beta|^{2}, \\
\alpha=\frac{z_{21}}{\left(z_{\mathrm{opt}}+z_{11}\right)}, \\
\beta=\frac{z_{22}^{*}}{\left(2 \operatorname{Re}\left(z_{22}\right)-\alpha z_{12}\right)},
\end{gathered}
$$

and Re denotes the real part. Observe that

$$
\mathbf{h}^{\mathrm{iid}}=\mathbf{D C M I} \mathbf{h}_{w}=\tau \mathbf{I}_{\S} \mathbf{h}_{w}
$$

such that the SNR is given by

$$
\gamma_{n}^{\mathrm{iid}}=|\tau|^{2} \frac{\varepsilon}{4 k T_{0} B \sigma^{2}} \max _{\mathbf{I}_{\delta}}\left\|\mathbf{I}_{\delta} \mathbf{h}_{w}\right\|^{2},
$$

where $|\tau|^{2}=\left(r_{\text {opt }} / r_{A}\right)|\alpha \beta|^{2}$. Comparing the two equations, we have

$$
N_{0}=\frac{4 k T_{0} B \sigma^{2}}{|\tau|^{2}}
$$

Hence, we normalize the noise covariance by $4 k T_{0} B \sigma^{2}$ and the output SNR by $|\tau|^{2}$ such that the normalized output SNR is given by

$$
\gamma_{n}^{o}=\frac{\mathcal{E}}{N_{0}} \max _{\mathbf{I}_{\S}}\left\|\boldsymbol{\Sigma}_{\mathbf{n}}^{-1 / 2} \mathbf{h}\right\|^{2},
$$

where $\mathbf{h}$ is given by (18) and $\boldsymbol{\Sigma}_{\mathbf{n}}$ by (21). The performance is evaluated in terms of the outage SNR-the probability that the SNR falls below a threshold $\gamma_{0}$

$$
P_{\text {out }}=\operatorname{Pr}\left(\gamma_{n}^{o}<\gamma_{0}\right) \text {. }
$$




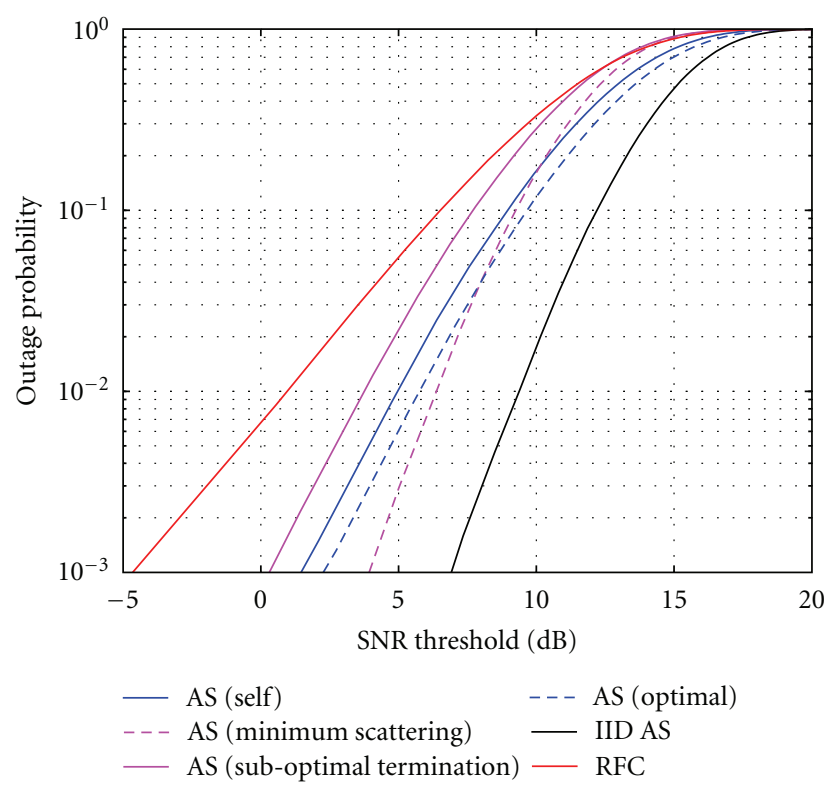

Figure 6: Traditional antenna selection (ULA): $(5,2)$.

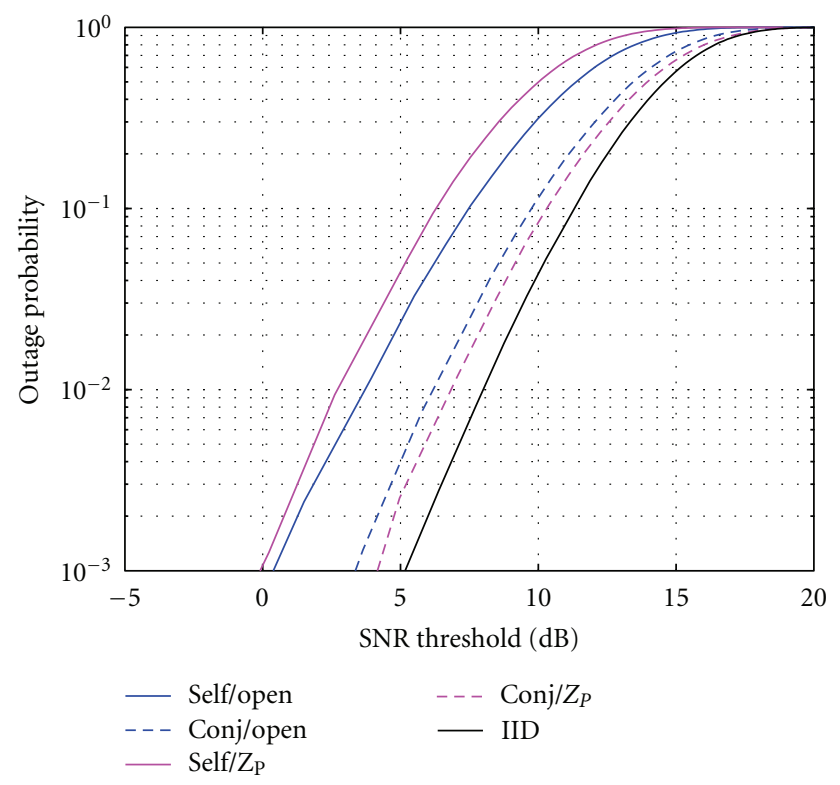

Figure 7: Parasitic antenna selection (UCA): $(4,2)$.

\section{Results}

We divide the results into two categories: uniform circular arrays and uniform linear arrays.

6.1. Uniform Linear Array. We begin by presenting the results for traditional antenna selection scheme for a ULA, where the inactive elements are left open circuited. The active elements are terminated into optimal or suboptimal matching networks derived in Section 4.2. Figure 6 shows the outage SNR results with the following settings: $N=5, L=$ $2, L_{r}=\lambda / 2$. Of practical importance is the outage level set at $1 \%$. We begin by observing that an $\operatorname{IID}(5,2)$ antenna selection system (denoted by IID AS), represents an upper bound on AS performance with large separation between the receive antennas. It offers a diversity gain of $10-\mathrm{dB}$ over a 2 -antenna diversity system separated by $\lambda / 2$ (denoted by RFC, c.f. Figure 1(b)). This is expected given the degrees of freedom added by antenna selection over a diversity system for the same number of RF chains.

The antenna selection results with the coupling and fading correlation notated by AS (self) and AS (optimal) denote the choice of active element matching network. The curves show that antenna selection in compact arrays provides a 3-4 dB improvement over conventional RFC systems, at a $1 \%$ outage. The performance, however, is still far from IID case even with optimal matching. The results for the configuration (c) in Figure 1 lie in between the self and optimal matching and are omitted for clarity. It clearly demonstrates that antenna selection is, in fact, preferred over employing all of the available antennas for compact arrays.

For sake of completeness, we also include results with the model assumed in the earlier studies on antenna selection. The curves reproduced with these models are shown in Figure 6 marked as minimum scattering and suboptimal termination model, respectively. As can be seen, the results as per [12] show optimistic performance (around $2 \mathrm{~dB}$ ), while, as per [13], antenna selection performs poorly (around $2 \mathrm{~dB}$ ) compared to a full-complexity system. This clearly underscores the importance of inactive element modeling and the choice of termination.

6.2. Uniform Circular Array. Next we present results for a UCA with $N=4, L=2, D_{r}=0.3 \lambda$ in Figure 7. It shows the outage SNR performance of antenna selection with optimal (denoted by Conj) or suboptimal (denoted by self) matching for active elements, and open-circuited inactive element termination (denoted by open) or optimal parasitic network (denoted by $Z_{P}$ ). The legend entries in the figure denote active element termination followed by inactive element termination separated by "/". Also shown is the curve with IID AS $(4,2)$.

The traditional antenna selection with optimal matching (denoted by Conj/Open) provides a 3-4 dB improvement over its self-matching counterpart (denoted by self/open). However, the optimal matching with optimal parasitic network (denoted by Conj $/ Z_{P}$ ) only offers a fraction of a $\mathrm{dB}$ improvement. The self-matching with optimal $\mathbf{Z}_{P}$ is the worst simply because $\mathbf{Z}_{P}$ has been optimized for the optimal matching network $\mathbf{Z}_{M}$ and is included for the sake of completeness. Also note that, unlike ULAs, optimalmatching by itself provides a significant improvement over self-matching.

The optimal parasitic networks considered so far have all been subset-dependent (i.e., depend on which set of antennas is active), where as the suboptimal ones are not. However, both types of networks are independent of the fading conditions. In the next section, we present a simpler parasitic scheme that adapts to the varying fading conditions. 


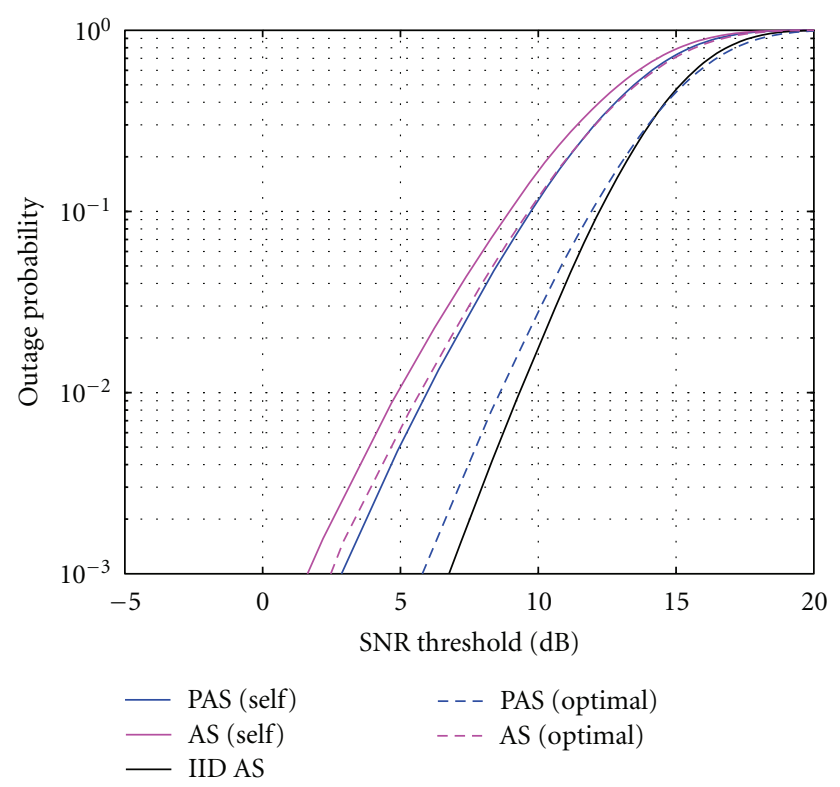

FIgURE 8: Parasitic switching (ULA): $(5,2)$.

\section{Parasitic Switching Strategies}

In this section, we provide a suboptimal design for the parasitic network that not only depends on the active subset but also the channel conditions. However, the implementation complexity is simplified by replacing multiport parasitic networks with two ports.

We propose a novel but simple strategy, in which each of the inactive element is terminated into an impedance $z_{p} \in$ $\mathcal{Z}_{\mathcal{P}}$, where $\mathcal{Z}_{\mathcal{P}}$ represents the set of all available terminations. The inactive element termination not only changes with the active subset but potentially may vary over time. Clearly, our ability to adaptively control the radiation pattern of the array lies in having a large set. However, as we shall see, even a small number of, but carefully chosen entries for $\mathfrak{Z}_{\mathcal{P}}$, can provide significant improvements.

We evaluate the performance of parasitic antenna selection system for $\mathcal{Z}_{\mathcal{P}}=\left\{0, \infty,-j x_{A}\right\}$, where 0 represents short-circuit, $\infty$ open-circuit, and $-j x_{A}$ a purely-reactive termination experimentally found to be the best fit for such a parasitic selection system, in the same sense as $z_{A}^{*}$ turns out to be an optimal match for active elements in the presence of mild coupling. This choice of $\mathcal{Z}_{\mathcal{P}}$ is simple and eliminates the need for a variable impedance under varying fading conditions. However, it turns out that due to directional sensitivity, the shorted parasitics do not offer much improvement, and this set can as well be limited to $\mathcal{Z}_{\mathcal{P}}=\left\{\infty,-j x_{A}\right\}$.

7.1. Parasitic Switching. Figure 8 shows the performance of the proposed parasitic antenna selection for $\operatorname{ULA}(5,2)$ denoted by PAS (self) and PAS (optimal) signifying the matching network $\mathbf{Z}_{M}$. For reference, curves from traditional antenna selection for $\operatorname{ULA}(5,2)$ are also included. It is interesting to note that parasitic switching with appropriate

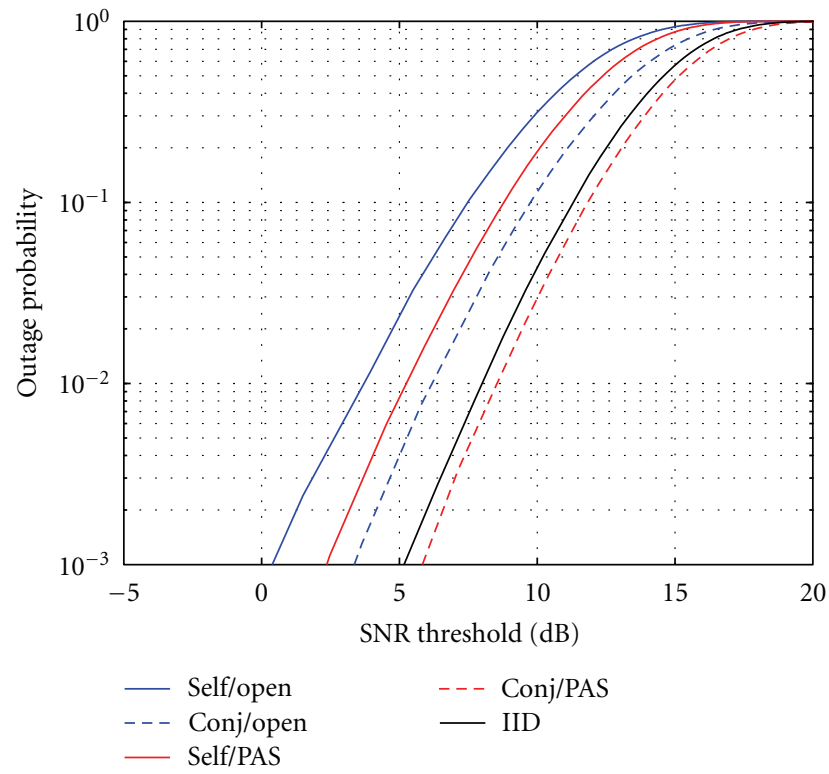

FIGURE 9: Parasitic switching (UCA): $(4,2)$.

matching achieves a performance close to that of an IID antenna selection system. At a $1 \%$ outage, optimal matching shows a $3 \mathrm{~dB}$ improvement over open-circuited antenna selection, once again highlighting how inactive element termination manifests itself in diversity gains.

Similar results are observed for a UCA $(4,2)$ (see Figure 9). However, it also shows an interesting result where antenna selection with optimal matching and parasitic switching performs better than an IID system. Several studies on multiantenna arrays with mutual coupling exhibit similar results in one form or the other, for example, $[14,28]$. The reason for such a behavior is generally attributed to the supergain phenomenon in antenna and microwave community. It primarily stems from the bandwidth assumptions of the system model. The matching and parasitic networks designed here are computed at the center frequency. However, such networks might have severe bandwidth limitations and to appropriately account for it, a broadband version of the problem must be investigated aimed at designing broadband matching networks [29].

7.2. Parasitic Selection Combining. A special case of this study surfaces when we consider selection combining (SC), that is, $L=1$. Unlike parasitic antenna selection (where $L>$ 1), parasitic selection combining (PSC) with self-matching registers a remarkable increase in the performance compared to its open-circuited counterpart, as shown in Figure 10 which shows the results for parasitic selection combining for a ULA of size $0.5 \lambda$.

One of the nifty things about this scheme is that as more antennas are packed in a fixed length array, the diversity gains relative to traditional selection become large. These gains however, diminish with increasing interelement spacings, as expected. It turns out that $\operatorname{PSC}(5,1)$ system performs just as well as PAS $(5,2)$ suggesting that we may as well do with 


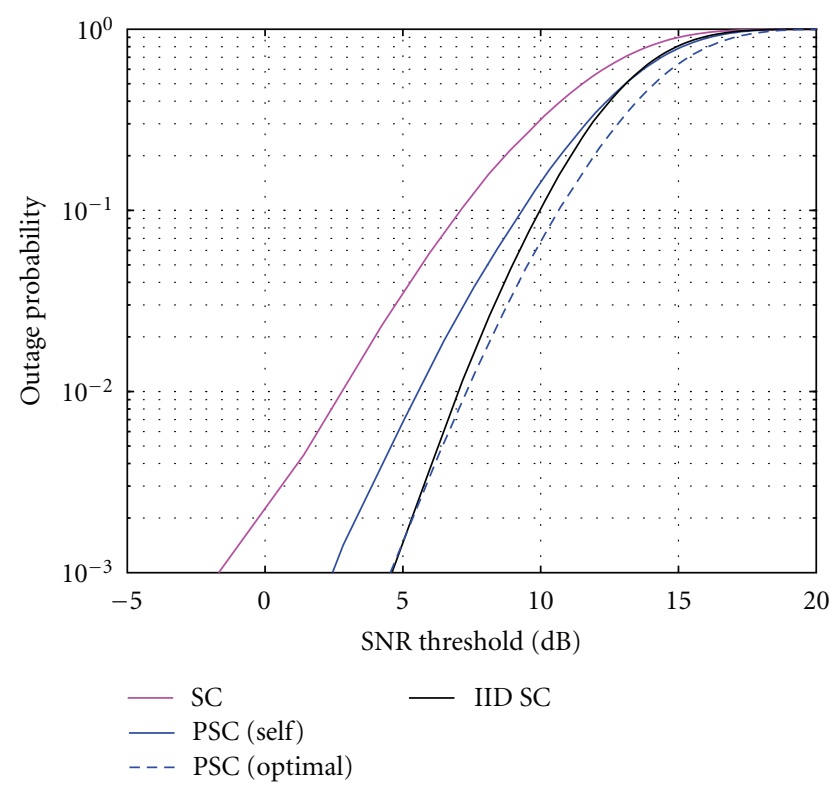

FIgURe 10: Parasitic selection combining (ULA): $(5,1)$.

just one RF chain thereby eliminating the need to build a multiport matching network.

Apparently, the loss incurred by employing one less RF chain is recovered by the availability of an additional parasitic element at the cost of an increased overhead as far as the number of parasitic switching states is concerned. It is important to point out that the active element is selectable, unlike ESPAR systems which employ a single active element with a set of parasitic elements arranged in a circular fashion around it.

7.3. Parasitic Switching with Fixed Active Elements. The performance benefits for any selection system come at the cost of an additional hardware-switching circuitry. Although, in selection systems with i.i.d. fading and noise, it suffices to pick the antenna subset with the $L$ highest SNR branches, such is not the case here due to coupling between the elements and correlated noise. As mentioned earlier, the switching network needs to cycle through $\left(\begin{array}{c}N \\ L\end{array}\right)$ subsets to arrive at the optimal configuration. Also, for the parasitic antenna selection scheme, the number of possible states for the inactive-element termination grows tremendously with the set $\mathcal{Z}_{\mathcal{P}}$ (given by $2^{N-L}$ in our case).

One drawback of antenna selection is the insertion loss arising due to the presence of switches in the direct RF signal paths. This loss typically ranges from a fraction of a $\mathrm{dB}$ to several $\mathrm{dB}[9]$, which, in our case, will ruin most of the benefits gained by employing any kind of selection in compact arrays. Although, certain RF MEMS switches [30] are known to offer less than $1 \mathrm{~dB}$ insertion loss at low speeds (switching time in $\mu s$ ), we explore the possibility of ruling out any insertion losses by avoiding switches in the direct RF path.



Figure 11: Parasitic switching w/o antenna selection (ULA): $(5,2)$.

We study parasitic switching without selection, that is, a predetermined set of elements (the ones that provide the optimal expected performance) is always active; however, the inactive elements are free to switch to any of the terminations available in $\mathcal{Z}_{\mathcal{P}}$. We consider two cases here: 2 RF chains for $\operatorname{AS}(5,2)$ (the far-end elements are always active) and $1 \mathrm{RF}$ chain for $\mathrm{SC}(5,1)$ (the middle element is always active).

The results are shown in Figure 11 (where the letter F in the legend entries represents "fixed"). It is interesting to note that this technique incurs a loss of only about $1 \mathrm{~dB}$ for both optimal and self-matching, while still retaining most of the benefits offered by the parasitic antenna selection. We make a special mention here that having the inactive elements as open circuited reduces to a conventional 2 element system. This strongly suggests that employing traditional antenna selection for compact arrays is definitely a suboptimal strategy and that parasitic switching even without selection can offer significant diversity gains.

\section{Discussion}

In the previous sections, we have seen how coupling can be exploited to improve the performance of antenna selection systems for compact arrays with appropriate matching networks. However, it is important to mention some of the challenges posed by the techniques presented here.

8.1. Optimal Matching Network. The optimal matching network shown here has certain limitations. While it provides the optimal performance for any given channel condition, multiport matching networks are nonrobust and known to shut down the RF bandwidth of the system [31], thereby 


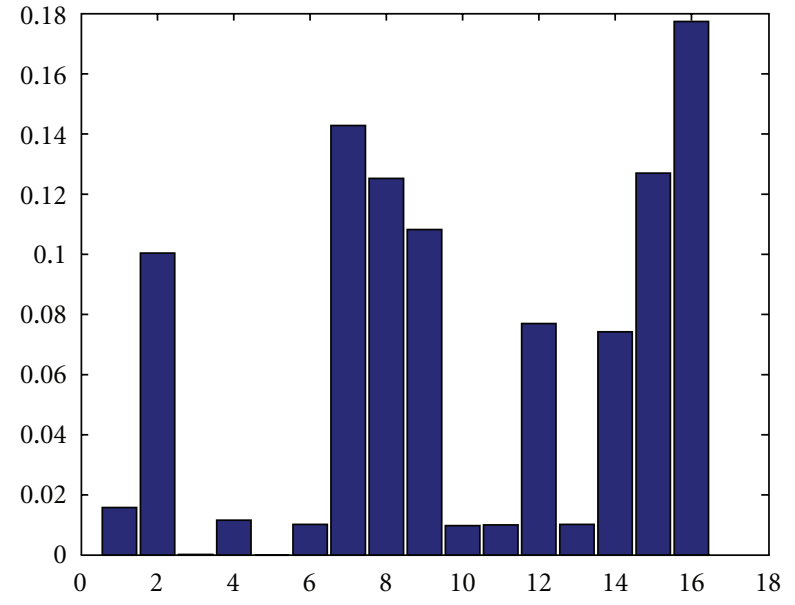

FiguRE 12: Histogram of parasitic switching combinations (ULA): $(5,2)$.

rendering it of little practical importance. The self-matching network on the other hand is robust but suboptimal, in that, it does not adapt to the varying channel conditions. Nevertheless, optimal matching serves as a benchmark for performance evaluation. We believe that an adaptive selfmatching network should offer a good tradeoff between the two.

8.2. Switching Aspects. We have already touched on the switching aspects in antenna selection systems. However, how often active subsets have to be chosen could be a significant overhead on the implementation costs. But in a slowfading or quasistatic channels like those of indoor/wireless LAN scenarios, the switching frequency is considerably reduced.

Another aspect is that of the associated parasitic switching states. Figure 12 shows the histogram of $\mathrm{Z}_{P}$ that maximizes the SNR at the receiver for $\operatorname{PSC}(5,1)$ system. It is evident that only about a half of the available combinations contribute to the overall performance, suggesting that a quick search procedure can be devised by ignoring the least likely candidates to tradeoff complexity with performance.

8.3. Impact of Inter-Element Spacing. As can be seen, the interelement spacing plays a vital role here. In [32], circular arrays with three possible terminations for the switched parasitic elements, namely, $0, \infty, z$ (where $z$ is a variable impedance) have been mentioned. However, for simulation purposes, only open and short terminations are considered. Unlike linear arrays, all the parasitic elements in a circular array are at an equal distance from the central driven element, and, thus, each parasitic element has the same amount of coupling. While such circular arrays have been studied in great detail over the past, little attention has been given to impedance other than short and open.
Besides, in the above mentioned studies, it has usually been considered that the open-circuit elements are transparent, which is a valid assumption for circular arrays with distances of the order of $0.2 \lambda$, and only the shorted elements arranged in a circular arc are known to regulate the directivity. However, this is not the case here. And the phenomenon in action here has a profound effect because the mutual coupling is stronger at close spacings. This is the reason why the gains realized from parasitic selection diminish for higher spacings.

\section{Conclusion}

We presented a detailed transceiver model for antenna selection applied to compact receive arrays which accounts for coupling among the elements and models the impact of inactive-element termination on system performance. We considered various design approaches for antenna selection strategies primarily for two types of uniform arrays-linear and circular. Apart from considering optimal designs for matching networks and parasitic networks (which may not be practically feasible from an implementation and/or cost perspective), we provided some simpler strategies that achieve near-optimal performance. We also considered designs where we only apply parasitic switching over a predefined set of elements in order to minimize losses arising due to RF switches.

Specifically, we showed that uniform linear arrays could in fact benefit from selection over using all of the available antennas, thereby, reducing the number of RF chains and saving power and cost. The performance improvement, though, depends on the spacing (i.e., amount of coupling in the system). For circular arrays, we showed that the optimal matching networks can easily be implemented by use of Butler matrices since the actual matching reduces to that of two-port networks (or self-matching). Based on the space and cost constraints, different array geometries can be designed and employed differently as shown in this paper.

Simulations results reveal how important it is to model the transceiver and account for noise correlation in the RF chain. Besides, it is vital to model the inactive elements as scatterers for close spacings and that wrong assumptions could result in misleading conclusions.

We conclude that different inactive-element terminations can impact performance in profoundly different ways for different array configurations. It turned out that such a system even with a single RF chain can deliver a huge performance improvement, thereby, making the hardware much simpler and power efficient. The overheads involved with this system like exhaustive search and possible insertion loss were provided alternatives with, which renders this approach as a lucrative option to apply in practical systems.

In a nutshell, antenna selection offers a distinct advantage in portable or handheld devices due to significant coupling among the antennas. Thus, packing more antennas in a constrained space and employing appropriate selection scheme can profoundly improve the system performance. 


\section{Appendices}

\section{A. Equivalent Channel}

Observe that we can rewrite (20) as

$$
\begin{aligned}
& \mathbf{C}=\mathbf{Q} \underbrace{\left(z_{21}\left(\boldsymbol{\Lambda}_{\S}^{\prime}+z_{11} \mathbf{I}\right)^{-1}\right)}_{\boldsymbol{\Lambda}_{C}} \mathbf{Q}^{H}, \\
& \mathbf{D}=\mathbf{Q}\left(z_{l}\left[\left(z_{l}+z_{22}\right) \mathbf{I}-z_{12} \boldsymbol{\Lambda}_{C}\right]^{-1}\right) \mathbf{Q}^{H}, \\
& \mathbf{M}=\mathbf{Q}\left(\boldsymbol{\Lambda}_{a s}\left(\boldsymbol{\Lambda}_{\S}+\boldsymbol{\Lambda}_{s s}\right)^{-1}\right) \mathbf{Q}^{H} .
\end{aligned}
$$

The equivalent selection matrix (11) can similarly be broken into

$$
\mathbf{T}_{\S}=\mathbf{I}_{\S}-\mathbf{Q} \boldsymbol{\Lambda}_{\S \bar{\delta}}\left(\boldsymbol{\Lambda}_{P}+\boldsymbol{\Lambda}_{\bar{\gamma} \bar{\delta}}\right)^{-1} \mathbf{Q}^{H} \mathbf{I}_{\bar{\gamma}},
$$

where $\mathbf{D}=\mathbf{Q} \boldsymbol{\Lambda}_{D} \mathbf{Q}^{H}$ and $\mathbf{M}=\mathbf{Q} \boldsymbol{\Lambda}_{M} \mathbf{Q}^{H}$. From optimal matching, we note that

$$
\Lambda_{\delta}+\Lambda_{s s}=\operatorname{Re}\left[\Lambda_{\delta}\right], \quad \Lambda_{\delta}^{\prime}=z_{\text {opt }} \mathbf{I}
$$

implying that $\boldsymbol{\Lambda}_{C}$ and $\boldsymbol{\Lambda}_{D}$ are independent of $\mathbf{Z}_{P}$, while

$$
\begin{aligned}
\boldsymbol{\Lambda}_{M} & =\sqrt{r_{\mathrm{opt}}}\left(\operatorname{Re}\left[\boldsymbol{\Lambda}_{\delta}\right]\right)^{-1 / 2} \\
& =\sqrt{r_{\mathrm{opt}}}\left(\operatorname{Re}\left[\boldsymbol{\Lambda}_{\delta \&}-\boldsymbol{\Lambda}_{\delta \bar{\delta}}\left(\boldsymbol{\Lambda}_{P}+\boldsymbol{\Lambda}_{\bar{\delta} \bar{\delta}}\right)^{-1} \boldsymbol{\Lambda}_{\bar{\delta}}\right]\right)^{-1 / 2} .
\end{aligned}
$$

The equivalent fading path-gains can thus be written as

$$
\begin{aligned}
\mathbf{h} & =\mathbf{Q} \boldsymbol{\Lambda}_{D} \boldsymbol{\Lambda}_{C} \boldsymbol{\Lambda}_{M} \mathbf{Q}^{H}\left(\mathbf{T}_{\S} \mathbf{h}_{o}\right) \\
& =\mathbf{Q} \boldsymbol{\Lambda}_{D} \boldsymbol{\Lambda}_{C} \boldsymbol{\Lambda}_{M} \mathbf{h}^{\prime},
\end{aligned}
$$

and the noise covariance for amplifier-noise dominant scenarios is given by

$$
\begin{aligned}
\boldsymbol{\Sigma}_{\mathbf{n}}^{\prime} & =\mathbf{D C}\left[r_{a} \mathbf{I}+g_{a}\left(\mathbf{Z}_{s}^{\prime}+z_{\mathrm{cor}} \mathbf{I}\right)\left(\mathbf{Z}_{s}^{\prime}+z_{\mathrm{cor}} \mathbf{I}\right)^{H}\right] \mathbf{C}^{H} \mathbf{D}^{H} \\
& =\left|\sigma_{a}\right|^{2} \mathbf{Q} \boldsymbol{\Lambda}_{D} \boldsymbol{\Lambda}_{C} \boldsymbol{\Lambda}_{C}^{H} \boldsymbol{\Lambda}_{D}^{H} \mathbf{Q}^{H},
\end{aligned}
$$

where

$$
\left|\sigma_{a}\right|^{2}=r_{a}+g_{a}\left|z_{\mathrm{opt}}+z_{\mathrm{cor}}\right|^{2}
$$

\section{B. Optimal Parasitic Network Evaluation}

For $N=4$, let us consider an example active subset

$$
\mathbf{I}_{\delta}=\left[\begin{array}{llll}
1 & 0 & 0 & 0 \\
0 & 0 & 1 & 0
\end{array}\right]
$$

such that

$$
\begin{aligned}
\mathbf{Q}^{H} \mathbf{T}_{\S} & =\mathbf{Q}^{H} \mathbf{I}_{\S}-\boldsymbol{\Lambda}_{T} \mathbf{Q}^{H} \mathbf{I}_{\bar{\S}} \\
& =\frac{1}{\sqrt{2}}\left[\begin{array}{cccc}
1 & -\lambda_{T 1} & 1 & -\lambda_{T 1} \\
1 & -\lambda_{T 2} & -1 & \lambda_{T 2}
\end{array}\right],
\end{aligned}
$$

where we have used

$$
\mathbf{T}_{\S}=\mathbf{I}_{\S}-\mathbf{Q} \boldsymbol{\Lambda}_{T} \mathbf{Q}^{H} \mathbf{I}_{\bar{\gamma}}, \quad \boldsymbol{\Lambda}_{T}=\boldsymbol{\Lambda}_{\delta \bar{\delta}}\left(\boldsymbol{\Lambda}_{P}+\boldsymbol{\Lambda}_{\bar{\delta} \bar{\gamma}}\right)^{-1} .
$$

From

$$
\begin{aligned}
\mathbf{h}^{\prime} & =\mathbf{Q}^{H} \mathbf{T}_{\S} \mathbf{h}_{o} \\
& =\left(\mathbf{Q}^{H} \mathbf{I}_{\S}-\boldsymbol{\Lambda}_{T} \mathbf{Q}^{H} \mathbf{I}_{\S}\right) \mathbf{h}_{o} \\
& =\frac{1}{\sqrt{2}}\left[\begin{array}{cccc}
1 & -\lambda_{T 1} & 1 & -\lambda_{T 1} \\
1 & -\lambda_{T 2} & -1 & \lambda_{T 2}
\end{array}\right]\left[\begin{array}{l}
h_{1} \\
h_{2} \\
h_{3} \\
h_{4}
\end{array}\right]
\end{aligned}
$$

To illustrate, let us consider the first entry of $\mathbf{h}^{\prime}$

$$
h_{1}^{\prime}=\frac{1}{\sqrt{2}}\left(\left(h_{1}+h_{3}\right)-\lambda_{T 1}\left(h_{2}+h_{4}\right)\right),
$$

such that

$$
\begin{array}{r}
\mathrm{E}\left(\left|h_{1}^{\prime}\right|^{2}\right)=\frac{1}{2}\left(\mathrm{E}\left(\left|h_{1}+h_{3}\right|^{2}\right)+\left|\lambda_{T 1}\right|^{2} \mathcal{E}\left(\left|h_{2}+h_{4}\right|^{2}\right)\right. \\
\left.-2 \operatorname{Re}\left(\mathrm{E}\left(\left(h_{1}^{*}+h_{3}^{*}\right)\left(h_{2}+h_{4}\right)\right) \lambda_{T 1}\right)\right) \\
=\frac{1}{2}\left(\mu_{1}+\mu_{2}\left|\lambda_{T 1}\right|^{2}-2 \operatorname{Re}\left(\mu_{3} \lambda_{T 1}\right)\right)>0 .
\end{array}
$$

From

$$
\boldsymbol{\Lambda}_{P}=\arg _{\boldsymbol{\Lambda}_{P}} \max \left\{\sum_{k=1}^{L}\left|\left[\lambda_{M}\right]_{k}\right|^{2} \mathrm{E}\left(\left|h_{k}^{\prime}\right|^{2}\right)\right\},
$$

the eigen-values of the optimal parasitic network can be obtained by maximizing each term in the summation above individually, that is,

$$
\lambda_{P}=\arg \max _{x}\left\{\left|\lambda_{M}\right|^{2}\left(\mu_{1}+\mu_{2}\left|\lambda_{T}\right|^{2}-2 \operatorname{Re}\left(\mu_{3} \lambda_{T}\right)\right)\right\},
$$

where

$$
\begin{gathered}
\lambda_{T}=\lambda_{s \bar{s}}\left(j x+\lambda_{\bar{s} \bar{s}}\right)^{-1}, \\
\lambda_{M}=r_{o} \operatorname{Re}\left[\lambda_{s \&}-\lambda_{s \bar{s}}\left(j x+\lambda_{\bar{s} \bar{s}}\right)^{-1} \lambda_{\bar{s} s}\right]^{-1 / 2},
\end{gathered}
$$

and $\lambda_{P}=j x$ is the optimal reactive parasitic termination.

For a circular array with a full receive scattering, the fading path-gain covariance matrix $\boldsymbol{\Sigma}_{\mathbf{h}}$ has a circulant structure of the form

$$
\boldsymbol{\Sigma}_{\mathbf{h}}=\left[\begin{array}{cccc}
1 & \rho_{1} & \rho_{2} & \rho_{1} \\
\rho_{1} & 1 & \rho_{1} & \rho_{2} \\
\rho_{2} & \rho_{1} & 1 & \rho_{1} \\
\rho_{1} & \rho_{2} & \rho_{1} & 1
\end{array}\right] .
$$

Using Kronecker spatial-correlation model $\mathbf{h}_{o}=\Sigma_{\mathbf{h}}^{1 / 2} \mathbf{h}_{w}$, explicit relationships between $\mu$ 's and $\rho$ 's can be calculated. Thus, the optimal values can be found by locating maxima of a polynomial of degree two. 


\section{References}

[1] D. G. Brennan, "Linear diversity combining techniques," Proceedings of the IEEE, vol. 91, no. 2, pp. 331-356, 2003.

[2] S. M. Alamouti, "A simple transmit diversity technique for wireless communications," IEEE Journal on Selected Areas in Communications, vol. 16, no. 8, pp. 1451-1458, 1998.

[3] G. J. Foschini and M. J. Gans, "On limits of wireless communications in a fading environment when using multiple antennas," Wireless Personal Communications, vol. 6, no. 3, pp. 311-335, 1998.

[4] P. W. Wolniansky, G. J. Foschini, G. D. Golden, and R. A. Valenzuela, "V-BLAST: an architecture for realizing very high data rates over the rich-scattering wireless channel," in Proceedings of the International Symposium on Signals, Systems and Electronics, pp. 295-300, 1998.

[5] N. Kong and L. B. Milstein, "Average SNR of a generalized diversity selection combining scheme," IEEE Communications Letters, vol. 3, no. 3, pp. 57-79, 1999.

[6] M. Z. Win and J. H. Winters, "Analysis of hybrid selection/maximal-ratio combining in rayleigh fading," IEEE Transactions on Communications, vol. 47, no. 12, pp. 1773 1776, 1999.

[7] A. Gorokhov, D. Gore, and A. Paulraj, "Receive antenna selection for MIMO flat-fading channels: theory and algorithms," IEEE Transactions on Information Theory, vol. 49, no. 10, pp. 2687-2696, 2003.

[8] M. Z. Win and J. H. Winters, "Virtual branch analysis of symbol error probability for hybrid selection/maximalratio combining in rayleigh fading," IEEE Transactions on Communications, vol. 49, no. 11, pp. 1926-1934, 2001.

[9] A. F. Molisch and M. Z. Win, "Mimo systems with antenna selection," IEEE Microwave Magazine, vol. 5, no. 1, pp. 46-56, 2004.

[10] R. Vaughan, "Switched parasitic elements for antenna diversity," IEEE Transactions on Antennas and Propagation, vol. 47, no. 2, pp. 399-405, 1999.

[11] R. F. Harrington, "Reactively controlled directive array," IEEE Transactions on Antennas and Propagation, vol. 26, no. 3, pp. 390-395, 1978.

[12] Z. Xu, S. Sfar, and R. S. Blum, "On the importance of modeling the mutual coupling for antenna selection for closely-spaced arrays," in Proceedings of the 40th Annual Conference on Information Sciences and Systems, pp. 1351-1355, March 2006.

[13] Y. Yang, S. Sfar, and R. S. Blum, "A simulation study of antenna selection for compact MIMO arrays," in Proceedings of the 42nd Annual Conference on Information Sciences and Systems, pp. 57-61, March 2008.

[14] C. P. Domizioli, B. L. Hughes, K. G. Gard, and G. Lazzi, "Receive diversity revisited: correlation, coupling and noise," in Proceedings of the 50th Annual IEEE Global Telecommunications Conference, pp. 3601-3606, Washington, DC, USA, November 2007.

[15] Y. Zhang, K. Hirasawa, and K. Fujimoto, "Opened parasitic elements nearby a driven dipole," IEEE Transactions on Antennas and Propagation, vol. 34, no. 5, pp. 711-713, 1986.

[16] H. Yagi, "Beam transmission of ultra short waves," Proceedings of the IEEE, vol. 16, pp. 715-741, 1928.

[17] M. Hamer and M. Butcher, "Experimental vehicular anglediversity antenna using mutual coupling," in Proceedings of the Antennas and Propagation Society International Symposium, pp. 1089-1091, Chicago, Ill, USA, July 1992.

[18] P. Mattheijssen, M. H. A. J. Herben, G. Dolmans, and L. Leyten, "Antenna-pattern diversity versus space diversity for use at handhelds," IEEE Transactions on Vehicular Technology, vol. 53, no. 4, pp. 1035-1042, 2004.

[19] C. A. Balanis, Antenna Theory: Analysis and Design, Wiley, 2nd edition, 1996.

[20] G. J. Burke and A. J. Poggio, "Numerica electromagnetics code (NEC)-method of moments," NOSC Technical Document 11, Ocean Systems Center, San Diego, Calif, USA, 1981.

[21] R. H. Clarke, "A statistical theory of mobile-radio reception," Bell Systems Technical Journal, vol. 47, pp. 957-1000, 1968.

[22] W. C. Jakes, Microwave Mobile Communications, IEEE Press, 1994.

[23] R. Q. Twiss, "Nyquist's and thevenin's theorems generalized for nonreciprocal linear networks," Journal of Applied Physics, vol. 26, no. 5, pp. 599-602, 1955.

[24] H. Rothe and W. Dahlke, "Theory of noisy fourpoles," Proceedings of the IRE, vol. 44, pp. 811-818, 1956.

[25] H. A. Haus and R. B. Adler, Circuit Theory of Linear Noisy Networks, Wiley, New York, NY, USA, 1959.

[26] C. P. Domizioli, B. L. Hughes, K. G. Gard, and G. Lazzi, "Optimal front-end design for MIMO receivers," in Proceedings of the IEEE Global Telecommunications Conference, pp. 37343739, New Orleans, La, USA, December 2008.

[27] http://datasheets.maxim-ic.com/en/ds/MAX2642-MAX2643. pdf.

[28] M. J. Gans, "Channel capacity between antenna arrayspart II: amplifier noise dominates," IEEE Transactions on Communications, vol. 54, no. 11, pp. 1983-1992, 2006.

[29] P. S. Taluja and B. L. Hughes, "Bandwidth limitations and broadband matching for coupled multi-antenna systems," in Proceedings of the IEEE Global Communications Conference, Houston, Tex, USA, 2011.

[30] E. R. Brown, "RF-MEMS switches for reconfigurable integrated circuits," IEEE Transactions on Microwave Theory and Techniques, vol. 46, no. 11, pp. 1868-1880, 1998.

[31] B. K. Lau, J. B. Andersen, G. Kristensson, and A. F. Molisch, "Impact of matching network on bandwidth of compact antenna arrays," IEEE Transactions on Antennas and Propagation, vol. 54, no. 11, part 1, pp. 3225-3238, 2006.

[32] N. L. Scott, "Diversity gain from a single-port adaptive antenna using switched parasitic elements illustrated with a wire and monopole prototype," IEEE Transactions on Antennas and Propagation, vol. 47, no. 6, pp. 1066-1070, 1999. 

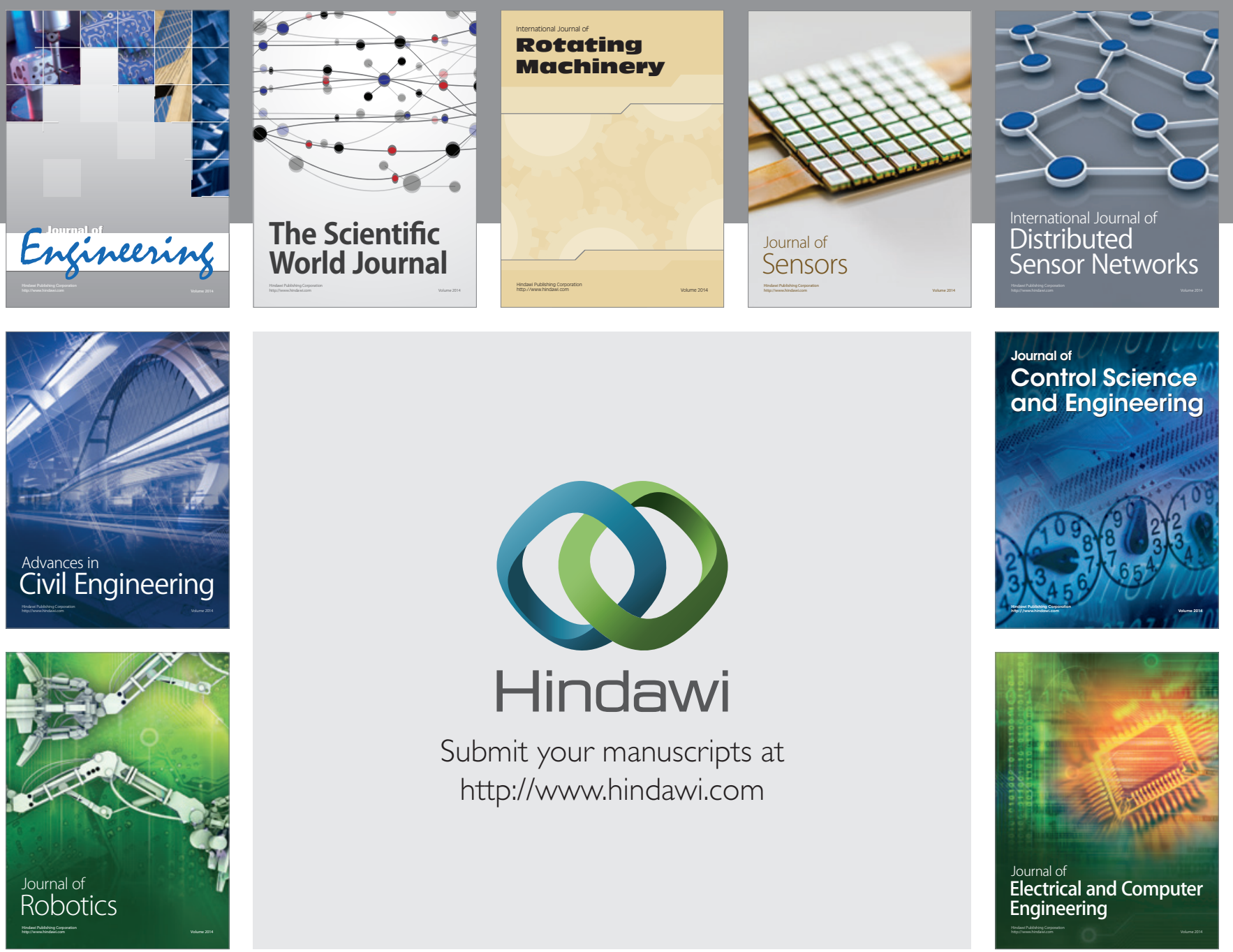

Submit your manuscripts at

http://www.hindawi.com
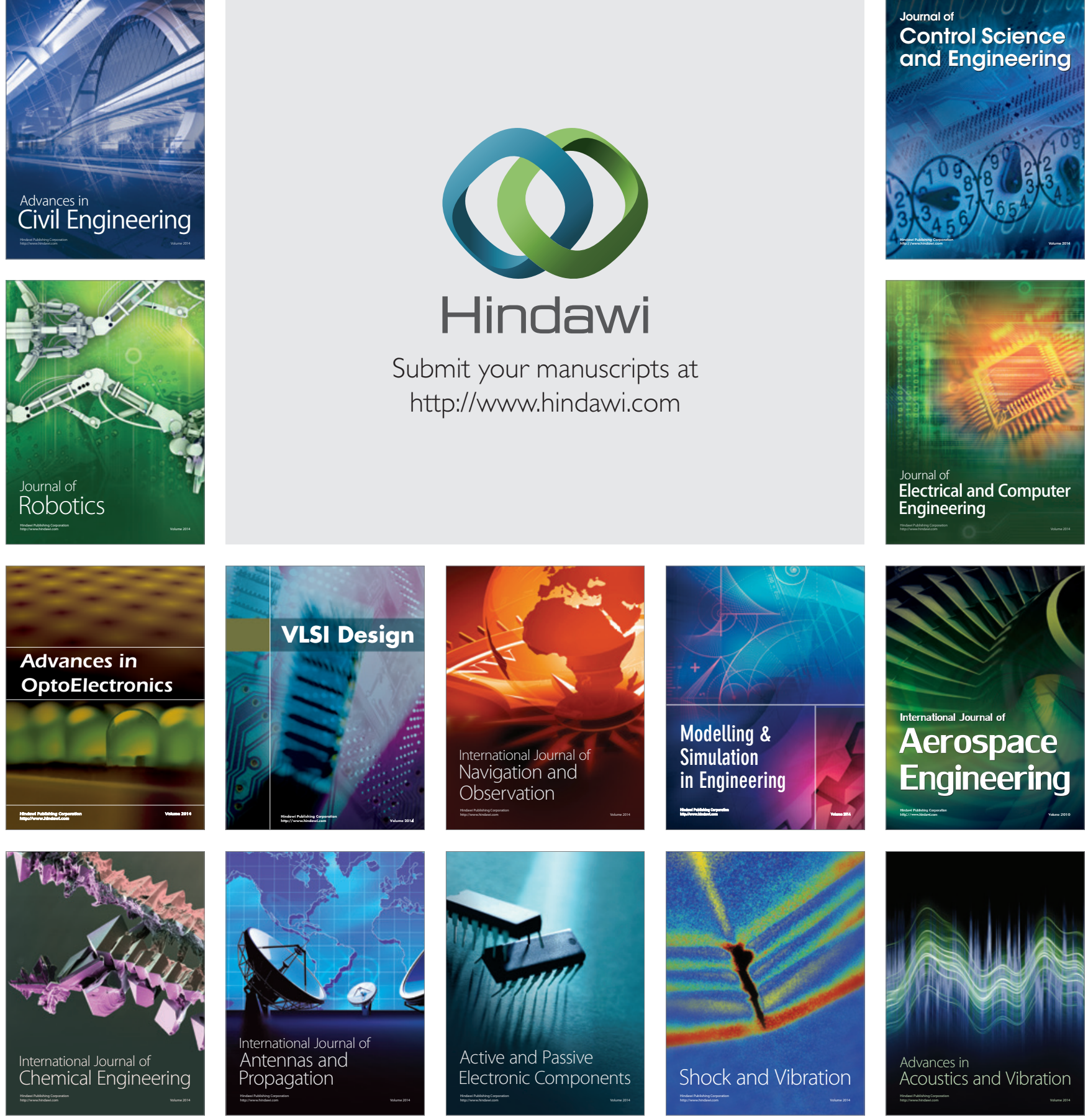